TRANSACTIONS OF THE

AMERICAN MATHEMATICAL SOCIETY

Volume 363, Number 2, February 2011, Pages 923-948

S 0002-9947(2010)05115-3

Article electronically published on September 29, 2010

\title{
GEVREY SOLUTIONS \\ OF THE IRREGULAR HYPERGEOMETRIC SYSTEM ASSOCIATED WITH AN AFFINE MONOMIAL CURVE
}

\author{
M. C. FERNÁNDEZ-FERNÁNDEZ AND F. J. CASTRO-JIMÉNEZ
}

To Zoghman Mebkhout on the occasion of his 60th birthday

\begin{abstract}
We describe the Gevrey series solutions at singular points of the irregular hypergeometric system (GKZ system) associated with an affine monomial curve. We also describe the irregularity complex of such a system with respect to its singular support.
\end{abstract}

\section{INTRODUCTION}

We explicitly describe the Gevrey solutions of the hypergeometric system associated with an affine monomial curve in $\mathbb{C}^{n}$ by using $\Gamma$-series introduced by I.M. Gel'fand, M.I. Graev, M.M. Kapranov and A.V. Zelevinsky [8, [10] and also used by M. Saito, B. Sturmfels and N. Takayama 30 in a very useful and slightly different form. We use these $\Gamma$-series to study the Gevrey filtration of the irregularity complex, as defined by Z. Mebkhout in [25, Sec. 6], of the corresponding analytic hypergeometric $\mathcal{D}$-module with respect to its singular support. Here $\mathcal{D}$ is the sheaf of linear differential operators with holomorphic coefficients on $\mathbb{C}^{n}$.

A general hypergeometric system $M_{A}(\beta)$ is associated with a pair $(A, \beta)$ where $A=\left(a_{i j}\right)$ is an integer $d \times n$ matrix of full rank $d$ and $\beta$ is a parameter vector in $\mathbb{C}^{d}\left([8],[9,[10])\right.$. The system $M_{A}(\beta)$ is defined by the following system of linear partial differential equations in the unknown $\varphi$ :

$$
\begin{gathered}
\prod_{i=1}^{n}\left(\frac{\partial}{\partial x_{i}}\right)^{u_{i}}(\varphi)-\prod_{i=1}^{n}\left(\frac{\partial}{\partial x_{i}}\right)^{v_{i}}(\varphi)=0 \quad\left(\text { for } u, v \in \mathbb{N}^{n}, A u=A v\right), \\
\sum_{j=1}^{n} a_{i j} x_{j} \frac{\partial \varphi}{\partial x_{j}}-\beta_{i} \varphi=0 \text { for } i=1, \ldots, d .
\end{gathered}
$$

If $d=1$ (i.e. if $A=\left(a_{1} a_{2} \cdots a_{n}\right)$ ) we say that the hypergeometric system $M_{A}(\beta)$ is associated with the affine monomial curve defined by $A$ in $\mathbb{C}^{n}$. If $d=2$

Received by the editors February 26, 2009 and, in revised form, May 15, 2009.

2000 Mathematics Subject Classification. Primary 32C38; Secondary 13N10, 14M25, 33C70.

The first author was partially supported by MTM2007-64509, FQM333 and by the FPU Grant AP2005-2360, MICINN (Spain).

The second author was partially supported by MTM2007-64509 and FQM333.

(C)2010 American Mathematical Society Reverts to public domain 28 years from publication 
and the vector $(1, \ldots, 1)$ is in the $\mathbb{Q}$-row span of $A$, then we say that $M_{A}(\beta)$ is associated with the projective monomial curve defined by $A$ in $\mathbb{P}_{n-1}(\mathbb{C})$.

Rational solutions of the hypergeometric system associated with a projective monomial curve have been studied in [6]. The holomorphic solutions of general hypergeometric systems at a generic point in $\mathbb{C}^{n}$ have been widely studied (see e.g. [9], 10], 1], 30], 27]). The rank of a general $M_{A}(\beta)$ is by definition the number of linearly independent holomorphic solutions of $M_{A}(\beta)$ at a generic point in $\mathbb{C}^{n}$. This rank equals, for generic $\beta \in \mathbb{C}^{d}$ and assuming $\mathbb{Z} A=\mathbb{Z}^{d}$, the normalized volume $\operatorname{vol}(\Delta)$ of the convex hull $\Delta$ of the columns of $A$ and the origin, considered as points in $\mathbb{R}^{d}$ (10 and 1$]$ ).

Several recent papers are devoted to the study of the exceptional parameters $\beta$, i.e. for which the rank jumps (see [23] and the references therein; see also [4]).

To set up the problem of computing the Gevrey solutions of a left $\mathcal{D}$-module, we will first recall the situation in the one-dimensional case.

Let $P=a_{m} \frac{d^{m}}{d x^{m}}+\cdots+a_{1} \frac{d}{d x}+a_{0}$ be an ordinary linear differential operator, of order $m$, with $a_{i}=a_{i}(x)$ a holomorphic function at the origin in $\mathbb{C}$. The slopes of $P$ are the slopes of the Newton polygon $N(P)$ of $P$ defined as the convex hull of the union of the quadrants $\left(i, i-v\left(a_{i}\right)\right)+\left(\mathbb{Z}_{\leq 0}\right)^{2}$ for $i=0, \ldots, m$, where $v\left(a_{i}\right)$ is the multiplicity of the zero of $a_{i}(x)$ at $x=0$. By Fuchs' Theorem, $P$ is regular at $x=0$ if and only if $N(P)$ is the quadrant $\left(m, m-v\left(a_{m}\right)\right)+\left(\mathbb{Z}_{\leq 0}\right)^{2}$.

Malgrange-Komatsu's Comparison Theorem states that $P$ is regular at $x=0$ if and only if the solution space $\operatorname{Sol}(P ; \widehat{\mathcal{O}} / \mathcal{O})$ is zero $([16$, , 22] $)$. Here $P$ acts naturally on the quotient $\widehat{\mathcal{O}} / \mathcal{O}$, where $\widehat{\mathcal{O}}=\mathbb{C}[[x]]$ (resp. $\mathcal{O}=\mathbb{C}\{x\}$ ) is the ring of formal (resp. convergent) power series in the variable $x$. This solution space measures the irregularity of $P$ at $x=0$ and will be denoted $\operatorname{Irr}_{0}(P):=\operatorname{Sol}(P ; \widehat{\mathcal{O}} / \mathcal{O})$.

Moreover, the vector space $\operatorname{Irr}_{0}(P)$ is filtered by the so-called Gevrey solutions of the equation $P(u)=0$. Let us denote by $\widehat{\mathcal{O}}_{s} \subset \widehat{\mathcal{O}}$ the subring of Gevrey series of order less than or equal to $s$ (where $s \geq 1$ is a real number). A formal power series $f=\sum_{i} f_{i} x^{i} \in \widehat{\mathcal{O}}$ is in $\widehat{\mathcal{O}}_{s}$ if and only if the series $\rho_{s}(f):=\sum_{i}(i !)^{1-s} f_{i} x^{i}$ is convergent at $x=0$. The irregularity $\operatorname{Irr}_{0}(P)$ is filtered by the solution subspaces $\operatorname{Irr}_{0}^{(s)}(P):=\operatorname{Sol}\left(P ; \widehat{\mathcal{O}}_{s} / \mathcal{O}\right)$. By the Comparison Theorem of J.P. Ramis [28] the jumps in this filtration are in bijective correspondence with the slopes of $N(P)$ and the dimension of each $\operatorname{Irr}_{0}^{(s)}(P)$ can also be read on $N(P)$.

In higher dimensions, one has analogous results but the situation is much more involved. Let $X$ be a complex manifold of dimension $n \geq 1$ and let us denote by $\mathcal{O}_{X}$ (resp. $\mathcal{D}_{X}$ ) the sheaf of rings of holomorphic functions on $X$ (resp. of linear differential operators with holomorphic coefficients). The role of the previous equation $P(u)=0$ is played here by a maximally overdetermined system of linear partial differential equations on $X$, or intrinsically by a holonomic $\mathcal{D}_{X}$-module $\mathcal{M}$. Z. Mebkhout introduced in 25 the irregularity of $\mathcal{M}$ along a smooth hypersurface $Y \subset X$ as the complex of sheaves of vector spaces $\operatorname{Irr}_{Y}(\mathcal{M}):=\mathbb{R H o m}_{\mathcal{D}_{X}}\left(\mathcal{M}, \mathcal{Q}_{Y}\right)$, where $\mathcal{Q}_{Y}$ is the quotient $\mathcal{Q}_{Y}=\frac{\mathcal{O}_{\widehat{X \mid Y}}}{\mathcal{O}_{X \mid Y}}$ and $\mathcal{O}_{\widehat{X \mid Y}}$ is the formal completion of $\mathcal{O}_{X}$ with respect to $Y$. This irregularity is nothing but the solution complex of $\mathcal{M}$ with values in $\mathcal{Q}_{Y}$. Z. Mebkhout also introduced the irregularity of order $s$ of $\mathcal{M}$ along $Y$ as the solution complex of $\mathcal{M}$ with values in $\mathcal{Q}_{Y}(s)$, i.e. $\operatorname{Irr}_{Y}^{(s)}(\mathcal{M}):=$ $\mathbb{R} \mathcal{H o m}_{\mathcal{D}_{X}}\left(\mathcal{M}, \mathcal{Q}_{Y}(s)\right)$, where $\mathcal{Q}_{Y}(s)$ is the quotient of the Gevrey series $\mathcal{O}_{\widehat{X \mid Y}}(s)$ of order less than or equal to $s$ along $Y$ by the sheaf $\mathcal{O}_{X \mid Y}$. Z. Mebkhout proved 
25. that these irregularity complexes belong to $\operatorname{Per}\left(\mathbb{C}_{Y}\right)$, the abelian category of perverse sheaves on $Y$ and that $\operatorname{Irr}_{Y}^{(s)}(\mathcal{M})$ is an increasing filtration of the irregularity $\operatorname{Irr}_{Y}(\mathcal{M})$. A real number $s>1$ is said to be an analytic slope of $\mathcal{M}$ along $Y$ at a point $p \in Y$ if the inclusion $\operatorname{Irr}_{Y}^{\left(s^{\prime}\right)}(\mathcal{M}) \subset \operatorname{Irr}_{Y}^{(s)}(\mathcal{M})$ is strict in a neighborhood of $p \in Y$ for $1<s^{\prime}<s$.

On the other hand, Y. Laurent defined the algebraic slopes of $\mathcal{M}$ by using interpolations of the order filtration and the Malgrange-Kashiwara filtration along $Y$ [18. Y. Laurent also proved that these algebraic slopes form a finite set of rational numbers.

The Comparison Theorem of Y. Laurent and Z. Mebkhout [19] proves that the analytic and the algebraic slopes coincide. Although the slopes of a $\mathcal{D}_{X}$-module $\mathcal{M}$ can be computed by an algorithm when $\mathcal{M}$ is algebraic [2, computations of the irregularity $\operatorname{Irr}_{Y}^{(s)}(\mathcal{M})$ are very rare. More precisely, under some conditions on the matrix $A$, the slopes of a hypergeometric system $M_{A}(\beta)$ can be combinatorially described from the matrix $A$ ([32], see also [5], [11]).

In this paper we explicitly describe the irregularity $\operatorname{Irr}_{Y}^{(s)}(\mathcal{M})$ for any $s$ and for $\mathcal{M}=\mathcal{M}_{A}(\beta)$ the hypergeometric system associated with an affine monomial curve when the associated semigroup is positive. Here $Y$ is the singular support of the system.

The paper has the following structure. In Sections 1 and 2 we recall the definition of Gevrey series and some essential results, proved by Z. Mebkhout 25, about the irregularity complex $\operatorname{Irr}_{Y}(\mathcal{M})$ of a holonomic $\mathcal{D}$-module $\mathcal{M}$ with respect to a hypersurface $Y$ in a complex manifold. We also recall the Comparison Theorem of Y. Laurent and Z. Mebkhout for the algebraic and analytic slopes of a holonomic $\mathcal{D}$-module 19 .

Section 3 deals with the basic properties of the $\Gamma$-series associated with the pair $(A, \beta)$ following [8, [10, Sec. 1] and [30, Sec. 3.4].

Sections 4 and 5 are devoted to the computation of the cohomology of the ir-

regularity complex $\operatorname{Irr}_{Y}^{(s)}\left(\mathcal{M}_{A}(\beta)\right)$ of the hypergeometric system $\mathcal{M}_{A}(\beta)$ associated with a smooth monomial curve and with a general monomial curve respectively, at any point of the singular support $Y$ of the system. In both cases the associated semigroup is assumed to be positive.

We prove our results by reducing the number of variables, by using the restriction functor in $\mathcal{D}$-module theory, and then applying the results from the 2-dimensional case [7].

This paper can be considered as a natural continuation of [7], [5] and [11], and its results should be related to the ones of [33]. We have often used some essential results of the book [30 about solutions of hypergeometric systems. Some of our results are related to [26] and also to [21] and [12].

The authors would like to thank Ch. Berkesch, N. Takayama and J.M. Tornero for their useful comments. They also thank the referee for his interesting suggestions.

\section{Gevrey Series}

Let $X$ be a complex manifold of dimension $n \geq 1, \mathcal{O}_{X}$ (or simply $\mathcal{O}$ ) the sheaf of holomorphic functions on $X$ and $\mathcal{D}_{X}$ (or simply $\mathcal{D}$ ) the sheaf of linear differential operators with coefficients in $\mathcal{O}_{X}$. The sheaf $\mathcal{O}_{X}$ has a natural structure of a left 
$\mathcal{D}_{X}$-module. Let $Z$ be a hypersurface in $X$ with defining ideal $\mathcal{I}_{Z}$. We denote by $\mathcal{O}_{X \mid Z}$ the restriction to $Z$ of the sheaf $\mathcal{O}_{X}$ (and we will also denote by $\mathcal{O}_{X \mid Z}$ its extension by 0 on $X$ ). Recall that the formal completion of $\mathcal{O}_{X}$ along $Z$ is defined as

$$
\mathcal{O}_{\widehat{X \mid Z}}:=\lim _{\leftarrow} \mathcal{O}_{X} / \mathcal{I}_{Z}^{k}
$$

By definition $\mathcal{O}_{\widehat{X \mid Z}}$ is a sheaf on $X$ supported on $Z$ and has a natural structure of a left $\mathcal{D}_{X}-$ module. We will also denote by $\mathcal{O}_{\widehat{X \mid Z}}$ the corresponding sheaf on $Z$. We denote by $\mathcal{Q}_{Z}$ the quotient sheaf defined by the following exact sequence:

$$
0 \rightarrow \mathcal{O}_{X \mid Z} \longrightarrow \mathcal{O}_{\widehat{X \mid Z}} \longrightarrow \mathcal{Q}_{Z} \rightarrow 0 \text {. }
$$

The sheaf $\mathcal{Q}_{Z}$, then has a natural structure of a left $\mathcal{D}_{X}-$ module.

Remark 1.1. If $X=\mathbb{C}$ and $Z=\{0\}$, then $\mathcal{O}_{\widehat{X \mid Z, 0}}$ is nothing but $\mathbb{C}[[x]]$, the ring of formal power series in one variable $x$, while $\mathcal{O}_{\widehat{X \mid Z}, p}=0$ for any nonzero $p \in X$. In this case $\mathcal{Q}_{Z, 0}=\frac{\mathbb{C}[[x]]}{\mathbb{C}\{x\}}$ and $\mathcal{Q}_{Z, p}=0$ for $p \neq 0$.

Definition 1.2. Assume $Y \subset X$ is a smooth hypersurface and that around a point $p \in X$ the hypersurface $Y$ is locally defined by $x_{n}=0$ for some system of local coordinates $\left(x_{1}, \ldots, x_{n}\right)$. Let us consider a real number $s \geq 1$. A germ $f=\sum_{i \geq 0} f_{i}\left(x_{1}, \ldots, x_{n-1}\right) x_{n}^{i} \in \mathcal{O}_{\widehat{X \mid Y}, p}$ is said to be a Gevrey series of order $s$ (along $Y$ at the point $p$ ) if the power series

$$
\rho_{s}(f):=\sum_{i \geq 0} \frac{1}{i !^{s-1}} f_{i}\left(x_{1}, \ldots, x_{n-1}\right) x_{n}^{i}
$$

is convergent at $p$.

The sheaf $\mathcal{O}_{\widehat{X \mid Y}}$ admits a natural filtration by the subsheaves $\mathcal{O}_{\widehat{X \mid Y}}(s)$ of Gevrey series of order $s, 1 \leq s \leq \infty$, where by definition $\mathcal{O}_{\widehat{X \mid Y}}(\infty)=\mathcal{O}_{\widehat{X \mid Y}}$. So we have $\mathcal{O}_{\widehat{X \mid Y}}(1)=\mathcal{O}_{X \mid Y}$. We can also consider the induced filtration on $\mathcal{Q}_{Y}$, i.e. the filtration by the subsheaves $\mathcal{Q}_{Y}(s)$ defined by the exact sequence:

$$
0 \rightarrow \mathcal{O}_{X \mid Y} \longrightarrow \mathcal{O}_{\widehat{X \mid Y}}(s) \longrightarrow \mathcal{Q}_{Y}(s) \rightarrow 0 .
$$

Definition 1.3. Let $Y$ be a smooth hypersurface in $X=\mathbb{C}^{n}$ and let $p$ be a point in $Y$. The Gevrey index of a formal power series $f \in \mathcal{O}_{\widehat{X \mid Y}, p}$ with respect to $Y$ is the smallest $1 \leq s \leq \infty$ such that $f \in \mathcal{O}_{\widehat{X \mid Y}}(s)_{p}$.

\section{IRREgularity COMPLEX AND SLOPES}

Let $X$ be a complex manifold. Here we recall the definition of the irregularity (also called the irregularity complex) of a left coherent $\mathcal{D}_{X}$-module given by Z. Mebkhout [25, (2.1.2) and page 98].

Recall that if $\mathcal{M}$ is a coherent left $\mathcal{D}_{X}$-module and $\mathcal{F}$ is any $\mathcal{D}_{X}$-module, the solution complex of $\mathcal{M}$ with values in $\mathcal{F}$ is by definition the complex

$$
\mathbb{R}_{\mathcal{H}} \mathrm{m}_{\mathcal{D}_{X}}(\mathcal{M}, \mathcal{F})
$$

which is an object of $D^{b}\left(\mathbb{C}_{X}\right)$, the derived category of bounded complexes of sheaves of $\mathbb{C}$-vector spaces on $X$. The cohomology sheaves of the solution complex are then $\mathcal{E} x t_{\mathcal{D}_{X}}^{i}(\mathcal{M}, \mathcal{F})$ (or simply $\mathcal{E} x t^{i}(\mathcal{M}, \mathcal{F})$ ) for $i \in \mathbb{N}$. 
Definition 2.1 ([25, (2.1.2) and page 98]). Let $Z$ be a hypersurface in $X$. The irregularity of $\mathcal{M}$ along $Z$ (denoted by $\operatorname{Irr}_{Z}(\mathcal{M})$ ) is the solution complex of $\mathcal{M}$ with values in $\mathcal{Q}_{Z}$, i.e.

$$
\operatorname{Irr}_{Z}(\mathcal{M}):=\mathbb{R} \mathcal{H} \operatorname{com}_{\mathcal{D}_{X}}\left(\mathcal{M}, \mathcal{Q}_{Z}\right) .
$$

If $Y$ is a smooth hypersurface in $X$ we also have the following definition (see 25. Déf. 6.3.7]).

Definition 2.2. For each $1 \leq s \leq \infty$, the irregularity of order $s$ of $\mathcal{M}$ with respect to $Y$ is the complex $\operatorname{Irr}_{Y}^{(s)}(\mathcal{M}):=\mathbb{R} \mathcal{H} o m_{\mathcal{D}_{X}}\left(\mathcal{M}, \mathcal{Q}_{Y}(s)\right)$.

Remark 2.3. Since $\mathcal{O}_{\widehat{X \mid Y}}(\infty)=\mathcal{O}_{\widehat{X \mid Y}}$ we have $\operatorname{Irr}_{Y}^{(\infty)}(\mathcal{M})=\operatorname{Irr}_{Y}(\mathcal{M})$. The support of the irregularity of $\mathcal{M}$ along $Z$ (resp. $\operatorname{Irr}_{Y}^{(s)}(\mathcal{M})$ ) is contained in $Z$ (resp. in $Y$ ).

If $X=\mathbb{C}, Z=\{0\}$ and $\mathcal{M}=\mathcal{D}_{X} / \mathcal{D}_{X} P$ is the $\mathcal{D}_{X}$-module defined by some nonzero linear differential operator $P\left(x, \frac{d}{d x}\right)$ with holomorphic coefficients, then $\operatorname{Irr}_{Z}(\mathcal{M})$ is represented by the complex

$$
0 \longrightarrow \frac{\mathbb{C}[[x]]}{\mathbb{C}\{x\}} \stackrel{P}{\rightarrow} \frac{\mathbb{C}[[x]]}{\mathbb{C}\{x\}} \longrightarrow 0
$$

where $P$ acts naturally on the quotient $\frac{\mathbb{C}[[x]]}{\mathbb{C}\{x\}}$.

Z. Mebkhout has proved [25, Th. 6.3.3] that for any holonomic $\mathcal{D}_{X}$-module $\mathcal{M}$ the complex $\operatorname{Irr}_{Y}^{(s)}(\mathcal{M})$ is a perverse sheaf on the smooth hypersurface $Y \subset X$ for any $1 \leq s \leq \infty$.

A complex $\mathcal{F}^{\bullet} \in D^{b}\left(\mathbb{C}_{X}\right)$ of sheaves of vector spaces is said to be constructible if there exists a stratification $\left(X_{\lambda}\right)$ of $X$ such that the cohomology sheaves $\mathcal{H}^{i}\left(\mathcal{F}^{\bullet}\right)$ are local systems on each $X_{\lambda}$. A constructible complex $\mathcal{F}^{\bullet}$ satisfies the support condition on $X$ if the following conditions hold:

(1) $\mathcal{H}^{i}\left(\mathcal{F}^{\bullet}\right)=0$ for $i<0$ and $i>n=\operatorname{dim}(X)$.

(2) The dimension of the support of $\mathcal{H}^{i}\left(\mathcal{F}^{\bullet}\right)$ is less than or equal to $n-i$ for $0 \leq i \leq n$.

A constructible complex $\mathcal{F}^{\bullet}$ is said to be perverse on $X$ (or even a perverse sheaf on $X$ ) if both $\mathcal{F}^{\bullet}$ and its dual $\mathbb{R} \mathcal{H} m_{\mathbb{C}_{X}}\left(\mathcal{F}^{\bullet}, \mathbb{C}_{X}\right)$ satisfy the support condition.

The category $\operatorname{Per}\left(\mathbb{C}_{X}\right)$ of perverse sheaves on $X$ is an abelian category (see [3]).

Remark 2.4. From [25, Cor. 6.3.5] each $\operatorname{Irr}_{Y}^{(s)}(-)$ for $1 \leq s \leq \infty$ is an exact functor from the category of holonomic $\mathcal{D}_{X}$-modules to the category of perverse sheaves on $Y$.

Moreover, the sheaves $\operatorname{Irr}_{Y}^{(s)}(\mathcal{M}), 1 \leq s \leq \infty$, form an increasing filtration of $\operatorname{Irr}_{Y}^{(\infty)}(\mathcal{M})=\operatorname{Irr}_{Y}(\mathcal{M})$. This filtration is called the Gevrey filtration of $\operatorname{Irr}_{Y}(\mathcal{M})$ (see [25, Sec. 6]).

Let us denote by

$$
\operatorname{Gr}_{s}\left(\operatorname{Irr}_{Y}(\mathcal{M})\right):=\frac{\operatorname{Irr}_{Y}^{(s)}(\mathcal{M})}{\operatorname{Irr}_{Y}^{(<s)}(\mathcal{M})}
$$

for $1 \leq s \leq \infty$ the graded object associated with the Gevrey filtration of the irregularity $\operatorname{Irr}_{Y}(\mathcal{M})$ (see [19, Sec. 2.4]).

We say, with [19, Sec. 2.4], that $1 \leq s<\infty$ is an analytic slope of $\mathcal{M}$ along $Y$ at a point $p \in Y$ if $p$ belongs to the closure of the support of $\operatorname{Gr}_{s}\left(\operatorname{Irr}_{Y}(\mathcal{M})\right)$. 
Y. Laurent (17, [18]) defines, in an algebraic way, the algebraic slopes of any coherent $\mathcal{D}_{X}$-module $\mathcal{M}$ along $Y$. These algebraic slopes can be algorithmically computed if the module $\mathcal{M}$ is defined by differential operators with polynomial coefficients [2].

In [19, Th. 2.5.3], Y. Laurent and Z. Mebkhout prove that for any holonomic $\mathcal{D}_{X}$-module the analytic and the algebraic slopes, with respect to any smooth hypersurface, coincide and that they are rational numbers. In [5] and [11 the slopes at the origin (with respect to any hyperplane in $\mathbb{C}^{n}$ ) of the hypergeometric system associated to any affine monomial curve are described. In [32, M. Schulze and U. Walther describe the slopes of any hypergeometric system with respect to any coordinate variety in $\mathbb{C}^{n}$ under some assumption on the semigroup associated with the system. For technical reasons the definition of slope given in [5] and [1] is slightly different from the one of Y. Laurent: a real number $-\infty \leq r \leq 0$ is called a slope in [5] and [11] if $\frac{r-1}{r}$ is an algebraic slope in the sense of Y. Laurent [18].

\section{Hypergeometric SYSTEMS AND $\Gamma$-SERIES}

Hypergeometric systems are defined on $X=\mathbb{C}^{n}$. We denote by $A_{n}(\mathbb{C})$ or simply $A_{n}$ the complex Weyl algebra of order $n$, i.e. the ring of linear differential operators with coefficients in the polynomial ring $\mathbb{C}[x]:=\mathbb{C}\left[x_{1}, \ldots, x_{n}\right]$. The partial derivative $\frac{\partial}{\partial x_{i}}$ will be denoted by $\partial_{i}$.

Let $A=\left(a_{i j}\right)$ be an integer $d \times n$ matrix with rank $d$ and $\beta \in \mathbb{C}^{d}$. Let us denote by $E_{i}(\beta)$ for $i=1, \ldots, d$ the operator $E_{i}(\beta):=\sum_{j=1}^{n} a_{i j} x_{j} \partial_{j}-\beta_{i}$. The toric ideal $I_{A} \subset \mathbb{C}[\partial]:=\mathbb{C}\left[\partial_{1}, \ldots, \partial_{n}\right]$ associated with $A$ is generated by the binomials $\square_{u}:=\partial^{u_{+}}-\partial^{u_{-}}$for $u \in \mathbb{Z}^{n}$ such that $A u=0$ where $u=u_{+}-u_{-}$and $u_{+}, u_{-}$are both in $\mathbb{N}^{n}$ and have disjoint support.

The left ideal $A_{n} I_{A}+\sum_{i} A_{n} E_{i}(\beta) \subset A_{n}$ is denoted by $H_{A}(\beta)$, and it will be called the hypergeometric ideal associated with $(A, \beta)$. The (global) hypergeometric module associated with $(A, \beta)$ is by definition (see [8, [10]) the quotient $M_{A}(\beta):=$ $A_{n} / H_{A}(\beta)$.

When $X=\mathbb{C}^{n}$ is considered as a complex manifold, to the pair $(A, \beta)$ we can also associate the corresponding analytic hypergeometric $\mathcal{D}_{X}$-module, denoted by $\mathcal{M}_{A}(\beta)$, which is the quotient of $\mathcal{D}_{X}$ modulo the sheaf of left ideals in $\mathcal{D}_{X}$ generated by $H_{A}(\beta)$.

In what follows we will use $\Gamma$-series following $[8$ and $[10$, Sec. 1] and the way in which these objects are handled in [30, Sec. 3.4].

Let the pair $(A, \beta)$ be as before. Assume $v \in \mathbb{C}^{n}$. We will consider the $\Gamma$-series

$$
\varphi_{v}:=x^{v} \sum_{u \in L_{A}} \frac{1}{\Gamma(v+u+\mathbf{1})} x^{u} \in x^{v} \mathbb{C}\left[\left[x_{1}^{ \pm 1}, \ldots, x_{n}^{ \pm n}\right]\right]
$$

where $\mathbf{1}=(1,1, \ldots, 1) \in \mathbb{N}^{n}, L_{A}=\operatorname{ker}_{\mathbb{Z}}(A)$ and for $\gamma=\left(\gamma_{1}, \ldots, \gamma_{n}\right) \in \mathbb{C}^{n}$ one has by definition $\Gamma(\gamma)=\prod_{i=1}^{n} \Gamma\left(\gamma_{i}\right)$ (where $\Gamma$ is the Euler gamma function). Notice that the set $x^{v} \mathbb{C}\left[\left[x_{1}^{ \pm 1}, \ldots, x_{n}^{ \pm n}\right]\right]$ has a natural structure of a left $A_{n}(\mathbb{C})$-module although it is not a $\mathcal{D}_{X, 0}-$ module. Nevertheless, if $A v=\beta$, then the expression $\varphi_{v}$ formally satisfies the operators defining $\mathcal{M}_{A}(\beta)$. Let us notice that if $u \in L_{A}$, then $\varphi_{v}=\varphi_{v+u}$. 
If $v \in\left(\mathbb{C} \backslash \mathbb{Z}_{<0}\right)^{n}$, then the coefficient $\frac{1}{\Gamma(v+u+1)}$ is nonzero for all $u \in L_{A}$ such that $u_{i}+v_{i} \geq 0$ for all $i$ with $v_{i} \in \mathbb{N}$. We also have the following equality:

$$
\frac{\Gamma(v+\mathbf{1})}{\Gamma(v+u+\mathbf{1})}=\frac{(v)_{u_{-}}}{(v+u)_{u_{+}}}
$$

where for any $z \in \mathbb{C}^{n}$ and any $\alpha \in \mathbb{N}^{n}$ we have the convention

$$
(z)_{\alpha}=\prod_{i, \alpha_{i}>0} \prod_{j=0}^{\alpha_{i}-1}\left(z_{i}-j\right) .
$$

Following [30, pp. 132-133], the negative support of $v($ denoted by $\operatorname{nsupp}(v))$ is the set of indices $i$ such that $v_{i} \in \mathbb{Z}_{<0}$. We say that $v$ has minimal negative support if there is no $u \in L_{A}$ such that $\operatorname{nsupp}(v+u)$ is a proper subset of $\operatorname{nsupp}(v)$.

Assume $v \notin\left(\mathbb{C} \backslash \mathbb{Z}_{<0}\right)^{n}$ has minimal negative support. The negative support of $v$ is then a nonempty set and $\Gamma(v+\mathbf{1})=\infty$. Moreover for each $u \in L_{A}$ at least one coordinate of $v+u$ must be strictly negative (otherwise $\operatorname{nsupp}(v+u)=\emptyset \subsetneq$ $\operatorname{nsupp}(v))$. So $\Gamma(v+u+\mathbf{1})=\infty$ for all $u \in L_{A}$ and $\varphi_{v}=0$.

If $v \notin\left(\mathbb{C} \backslash \mathbb{Z}_{<0}\right)^{n}$ does not have minimal negative support, then there exists $u \in L_{A}$ such that $v+u$ has minimal negative support. If $\operatorname{nsupp}(v+u)=\emptyset$, then $\varphi_{v}=\varphi_{v+u} \neq 0$ while if $\operatorname{nsupp}(v+u) \neq \emptyset$, then $\varphi_{v}=\varphi_{v+u}=0$.

Following [30, pp. 132-133], for any $v \in \mathbb{C}^{n}$ we will consider the series

$$
\phi_{v}:=x^{v} \sum_{u \in N_{v}} \frac{(v)_{u_{-}}}{(v+u)_{u_{+}}} x^{u}
$$

where $N_{v}=\left\{u \in L_{A} \mid \operatorname{nsupp}(v+u)=\operatorname{nsupp}(v)\right\}$.

If $A v=\beta$, then $\phi_{v}$ is a solution of the hypergeometric ideal $H_{A}(\beta)$ (i.e. $\phi_{v}$ is formally annihilated by $\left.H_{A}(\beta)\right)$ if and only if $v$ has minimal negative support [30. Prop. 3.4.13].

For $v \in\left(\mathbb{C} \backslash \mathbb{Z}_{<0}\right)^{n}$ we have

$$
\frac{\Gamma(v+\mathbf{1})}{\Gamma(v+u+\mathbf{1})}=\frac{(v)_{u_{-}}}{(v+u)_{u_{+}}}
$$

and $\Gamma(v+\mathbf{1}) \varphi_{v}=\phi_{v}$.

If $v \notin\left(\mathbb{C} \backslash \mathbb{Z}_{<0}\right)^{n}$, then the coefficient of $x^{v}$ in $\phi_{v}$ is nonzero (in fact this coefficient is 1$)$ while it is zero in $\varphi_{v}$.

In order to simplify notation we will adopt in the sequel the following convention: for $v \in \mathbb{C}^{n}$ and $u \in L_{A}$ we will denote

$$
\Gamma[v ; u]:=\frac{(v)_{u_{-}}}{(v+u)_{u_{+}}}
$$

if $u \in N_{v}$ and $\Gamma[v ; u]:=0$ otherwise. With this convention we have

$$
\phi_{v}=x^{v} \sum_{u \in L_{A}} \Gamma[v ; u] x^{u} .
$$

In what follows we will describe the irregularity, along the singular locus, of the hypergeometric system associated with an affine monomial curve in $X=\mathbb{C}^{n}$ and with a parameter $\beta \in \mathbb{C}$. 


\section{The Case of a smooth monomial Curve}

Let $A=\left(1 a_{2} \cdots a_{n}\right)$ be an integer row matrix with $1<a_{2}<\cdots<a_{n}$ and $\beta \in \mathbb{C}$. Let us denote by $\mathcal{M}_{A}(\beta)$ the corresponding analytic hypergeometric system on $X=\mathbb{C}^{n}$. We will simply denote by $\mathcal{D}$ the sheaf $\mathcal{D}_{X}$ of linear differential operators with holomorphic coefficients.

Although it can be deduced from general results (see 8 and [1, Th. 3.9]), a direct computation shows in this case that the characteristic variety of $\mathcal{M}_{A}(\beta)$ equals $T_{X}^{*} X \cup T_{Y}^{*} X$, where $Y=\left(x_{n}=0\right)$. The module $\mathcal{M}_{A}(\beta)$ is then holonomic and its singular support is $Y$. Let us denote by $Z \subset \mathbb{C}^{n}$ the hyperplane $x_{n-1}=0$.

One of the main results in this section is

Theorem 4.1. Let $A=\left(\begin{array}{llll}1 & a_{2} & \cdots & a_{n}\end{array}\right)$ be an integer row matrix with $1<a_{2}<$ $\cdots<a_{n}$ and $\beta \in \mathbb{C}$. Then the cohomology sheaves of $\operatorname{Irr}_{Y}^{(s)}\left(\mathcal{M}_{A}(\beta)\right)$ satisfy:

i) $\mathcal{E}_{x} t_{\mathcal{D}}^{0}\left(\mathcal{M}_{A}(\beta), \mathcal{Q}_{Y}(s)\right)=0$ for $1 \leq s<a_{n} / a_{n-1}$.

ii) $\mathcal{E}_{x} t_{\mathcal{D}}^{0}\left(\mathcal{M}_{A}(\beta), \mathcal{Q}_{Y}(s)\right)_{\mid Y \cap Z}=0$ for $1 \leq s \leq \infty$.

iii) $\operatorname{dim}_{\mathbb{C}}\left(\mathcal{E} x t_{\mathcal{D}}^{0}\left(\mathcal{M}_{A}(\beta), \mathcal{Q}_{Y}(s)\right)_{p}\right)=a_{n-1}$ for all $s \geq a_{n} / a_{n-1}$ and $p \in Y \backslash Z$.

iv) $\mathcal{E}_{x} t_{\mathcal{D}}^{i}\left(\mathcal{M}_{A}(\beta), \mathcal{Q}_{Y}(s)\right)=0$ for $i \geq 1$ and $1 \leq s \leq \infty$.

The main ingredients in the proof of Theorem 4.1] are: Corollary 4.4, the corresponding results for the case of monomial plane curves [7], Cauchy-Kovalevskaya Theorem for Gevrey series (see [20, Cor. 2.2.4]), the perversity of $\operatorname{Irr}_{Y}^{(s)}\left(\mathcal{M}_{A}(\beta)\right)$ [25, Th. 6.3.3] and Kashiwara's Constructibility Theorem [13].

We will also describe a basis of the solution vector space in part iii) of Theorem 4.1 (see Theorem 4.23).

4.1. Reduction of the number of variables by restriction. In the sequel we will use some results concerning the restriction of hypergeometric systems.

Theorem 4.2 ([5, Th. 4.4]). Let $A=\left(1 a_{2} \cdots a_{n}\right)$ be an integer row matrix with $1<a_{2}<\cdots<a_{n}$ and $\beta \in \mathbb{C}$. Then for $i=2, \ldots, n$, the restriction of $\mathcal{M}_{A}(\beta)$ to $\left(x_{i}=0\right)$ is isomorphic to the $\mathcal{D}^{\prime}$-module

$$
\mathcal{M}_{A}(\beta)_{\mid\left(x_{i}=0\right)}:=\frac{\mathcal{D}}{\mathcal{D} H_{A}(\beta)+x_{i} \mathcal{D}} \cong \frac{\mathcal{D}^{\prime}}{\mathcal{D}^{\prime} H_{A^{\prime}}(\beta)},
$$

where $A^{\prime}=\left(\begin{array}{lllllll}1 & a_{2} & \cdots & a_{i-1} & a_{i+1} & \cdots & a_{n}\end{array}\right)$ and $\mathcal{D}^{\prime}$ is the sheaf of linear differential operators with holomorphic coefficients on $\mathbb{C}^{n-1}$ (with coordinates $x_{1}, \ldots, x_{i-1}, x_{i+1}$, $\left.\ldots, x_{n}\right)$.

Theorem 4.3. Let $A=(1 \mathrm{ka} k \mathrm{k})$ be an integer row matrix with $1 \leq a<b, 1<$ $k a<k b$ and $a, b$ relatively prime. Then for all $\beta \in \mathbb{C}$ there exist $\beta_{0}, \ldots, \beta_{k-1} \in \mathbb{C}$ such that the restriction of $\mathcal{M}_{A}(\beta)$ to $\left(x_{1}=0\right)$ is isomorphic to the $\mathcal{D}^{\prime}$-module

$$
\mathcal{M}_{A}(\beta)_{\mid\left(x_{1}=0\right)}:=\frac{\mathcal{D}}{\mathcal{D} H_{A}(\beta)+x_{1} \mathcal{D}} \simeq \bigoplus_{i=0}^{k-1} \mathcal{M}_{A^{\prime}}\left(\beta_{i}\right),
$$

where $\mathcal{D}^{\prime}$ is the sheaf of linear differential operators on the plane $\left(x_{1}=0\right)$ and $A^{\prime}=(a b)$. Moreover, for all but finitely many $\beta \in \mathbb{C}$ we can take $\beta_{i}=\frac{\beta-i}{k}$, $i=0,1, \ldots, k-1$.

An ingredient in the proof of Theorem 4.1 is the following. 
Corollary 4.4. Let $A=\left(\begin{array}{llll}1 & a_{2} & \cdots & a_{n}\end{array}\right)$ be an integer row matrix with $1<a_{2}<$ $\cdots<a_{n}$ and $\beta \in \mathbb{C}$. Then there exist $\beta_{i} \in \mathbb{C}, i=0, \ldots, k-1$ such that the restriction of $\mathcal{M}_{A}(\beta)$ to $X^{\prime}=\left(x_{1}=x_{2}=\cdots=x_{n-2}=0\right)$ is isomorphic to the $\mathcal{D}^{\prime}$-module

$$
\mathcal{M}_{A}(\beta)_{\mid X^{\prime}}:=\frac{\mathcal{D}}{\mathcal{D} H_{A}(\beta)+\left(x_{1}, x_{2}, \cdots, x_{n-2}\right) \mathcal{D}} \simeq \bigoplus_{i=0}^{k-1} \mathcal{M}_{A^{\prime}}\left(\beta_{i}\right),
$$

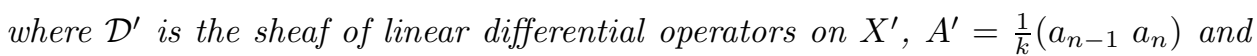
$k=\operatorname{gcd}\left(a_{n-1}, a_{n}\right)$. Moreover, for all but finitely many $\beta \in \mathbb{C}$ we can take $\beta_{i}=\frac{\beta-i}{k}$, $i=0,1, \ldots, k-1$.

Let us fix some notation and state some preliminary results in order to prove Theorem 4.3 .

Notation 4.5. Let $A$ be an integer $d \times n$-matrix of rank $d$ and $\beta \in \mathbb{C}^{n}$. For any weight vector $\omega \in \mathbb{R}^{n}$ and any ideal $J \subset \mathbb{C}[\partial]=\mathbb{C}\left[\partial_{1}, \ldots, \partial_{n}\right]$ we denote by $\operatorname{in}_{\omega}(J)$ the initial ideal of $J$ with respect to the graduation on $\mathbb{C}[\partial]$ induced by $w$. According to [30. p. 106] the fake initial ideal of $H_{A}(\beta)$ is the ideal $\operatorname{fin}_{\omega}\left(H_{A}(\beta)\right)=A_{n} \operatorname{in}_{\omega}\left(I_{A}\right)+$ $A_{n}(A \theta-\beta)$, where $\theta=\left(\theta_{1}, \ldots, \theta_{n}\right)$ and $\theta_{i}=x_{i} \partial_{i}$.

Now assume that $A=(1 \mathrm{kakb})$ is an integer row matrix with $1 \leq a<b$, $1<k a<k b$ and $a, b$ relatively prime.

Let us write $P_{1}=\partial_{2}^{b}-\partial_{3}^{a}, P_{2}=\partial_{1}^{k a}-\partial_{2}, P_{3}=\partial_{1}^{k b}-\partial_{3}$ and $E=\theta_{1}+k a \theta_{2}+$ $k b \theta_{3}-\beta$. It is clear that $P_{1} \in H_{A}(\beta)=\left\langle P_{2}, P_{3}, E\right\rangle \subset A_{3}$.

Let us fix $\omega=(1,0,0)$ until the end of this section.

Lemma 4.6. Let $A=(1 \mathrm{ka} k b)$ be an integer row matrix with $1 \leq a<b, 1<k a<$ $k b$ and $a, b$ relatively prime. Then

$$
\operatorname{fin}_{\omega}\left(H_{A}(\beta)\right)=A_{3} \operatorname{in}_{\omega}\left(I_{A}\right)+A_{3} E=A_{3}\left(P_{1}, E, \partial_{1}^{k}\right)
$$

for $\beta \notin \mathbb{N}^{*}:=\mathbb{N} \backslash\{0\}$ and for all $\beta \in \mathbb{N}^{*}$ big enough.

Proof. The proof follows from the application of Buchberger's algorithm in the Weyl algebra $A_{3}$ and the fact that $\partial_{1}^{k} \in \operatorname{fin}_{\omega}\left(H_{A}(\beta)\right)$ for $\beta$ as in the statement.

Definition 4.7 (30, Def. 5.1.1]). Let $I \subseteq A_{n}$ be a holonomic ideal (i.e. $A_{n} / I$ is holonomic) and $\widetilde{\omega} \in \mathbb{R}^{n} \backslash\{0\}$. The $b$-function $I$ with respect to $\widetilde{\omega}$ is the monic generator of the ideal

$$
\operatorname{in}_{(-\widetilde{\omega}, \widetilde{\omega})}(I) \cap \mathbb{C}[\tau],
$$

where $\tau=\widetilde{\omega}_{1} \theta_{1}+\cdots+\widetilde{\omega}_{n} \theta_{n}$ and $\operatorname{in}_{(-\widetilde{\omega}, \widetilde{\omega})}(I)$ is the initial ideal of $I$ with respect to the weight vector $(-\widetilde{\omega}, \widetilde{\omega})$.

Corollary 4.8. Let $A=(1 k a k b)$ be an integer row matrix with $1 \leq a<b$, $1<k a<k b$ and $a, b$ relatively prime. Then the $b$-function of $H_{A}(\beta)$ with respect to $\omega=(1,0,0)$ is

$$
b(\tau)=\tau(\tau-1) \cdots(\tau-(k-1))
$$

for all but finitely many $\beta \in \mathbb{C}$.

Proof. From [30, Th. 3.1.3] for all but finitely many $\beta \in \mathbb{C}$ we have

$$
\operatorname{in}_{(-\omega, \omega)}\left(H_{A}(\beta)\right)=\operatorname{fin}_{\omega}\left(H_{A}(\beta)\right) .
$$

Then by using Lemma 4.6 we get

$$
\operatorname{in}_{(-\omega, \omega)}\left(H_{A}(\beta)\right)=A_{3}\left(P_{1}, E, \partial_{1}^{k}\right)
$$


for all but finitely many $\beta \in \mathbb{C}$. An easy computation shows that $\left\{P_{1}, E, \partial_{1}^{k}\right\}$ is a Groebner basis of the ideal in $\operatorname{in}_{(-\omega, \omega)}\left(H_{A}(\beta)\right)$ with respect to any monomial order $>$ satisfying $\theta_{3}>\theta_{1}, \theta_{2}$ and $\partial_{2}^{b}>\partial_{3}^{a}$. In particular we can consider the lexicographic order

$$
x_{3}>x_{2}>\partial_{2}>\partial_{3}>x_{1}>\partial_{1},
$$

which is an elimination order for $x_{1}$ and $\partial_{1}$. So we get

$$
\operatorname{in}_{(-\omega, \omega)}\left(H_{A}(\beta)\right) \cap \mathbb{C}\left[x_{1}\right]\left\langle\partial_{1}\right\rangle=\left\langle\partial_{1}^{k}\right\rangle
$$

and since $x_{1}^{k} \partial_{1}^{k}=\theta_{1}\left(\theta_{1}-1\right) \cdots\left(\theta_{1}-(k-1)\right)$, we have

$$
\operatorname{in}_{(-\omega, \omega)}\left(H_{A}(\beta)\right) \cap \mathbb{C}\left[\theta_{1}\right]=\left\langle\theta_{1}\left(\theta_{1}-1\right) \cdots\left(\theta_{1}-(k-1)\right)\right\rangle .
$$

This proves the corollary.

Remark 4.9. Corollary 4.8 can be related to [5, Th. 4.3] which proves that for $A=\left(\begin{array}{llll}1 & a_{2} & \cdots & a_{n}\end{array}\right)$ with $1<a_{2}<\cdots<a_{n}$, the $b$-function of $H_{A}(\beta)$ with respect to $e_{i}$ is $b(\tau)=\tau$, for $i=2, \ldots, n$. Here $e_{i} \in \mathbb{R}^{n}$ is the vector with a 1 in the $i$-th coordinate and 0 elsewhere.

Recall (see e.g. [30, Def. 1.1.3]) that a Groebner basis of a left ideal $I \subset A_{n}$ with respect to $(-\omega, \omega) \in \mathbb{R}^{2 n}$ (or simply with respect to $\omega \in \mathbb{R}^{n}$ ) is a finite subset $G \subset I$ such that $I=A_{n} G$ and $\operatorname{in}_{(-\omega, \omega)}(I)=A_{n} \operatorname{in}_{(-\omega, \omega)}(G)$ where $\operatorname{in}_{(-\omega, \omega)}(G)=$ $\left\{\operatorname{in}_{(-\omega, \omega)}(P) \mid P \in G\right\}$.

Lemma 4.10. Let $A=(1 k a k b)$ be an integer row matrix with $1 \leq a<b$, $1<k a<k b$ and $a, b$ relatively prime. For all but finitely many $\beta \in \mathbb{C}$, a Groebner basis of $H_{A}(\beta) \subset A_{3}$ with respect to $\omega=(1,0,0)$ is

$$
\left\{P_{1}, P_{2}, P_{3}, E, R\right\}
$$

for some $R \in A_{3}$ satisfying $\operatorname{in}_{(-\omega, \omega)}(R)=\partial_{1}^{k}$.

The following proposition is a particular case of [4, Th. 6.5] (see also [29, Th. 2.1]) and it will be used later.

Proposition 4.11. Assume $A=\left(a_{1} a_{2} \cdots a_{n}\right)$ is an integer row matrix with $0<$ $a_{1}<a_{2}<\cdots<a_{n}$. For $\beta, \beta^{\prime} \in \mathbb{C}^{d}$ we have that $\mathcal{M}_{A}(\beta) \simeq \mathcal{M}_{A}\left(\beta^{\prime}\right)$ if and only if one of the following conditions holds:

i) $\beta, \beta^{\prime} \in \mathbb{N} A$.

ii) $\beta, \beta^{\prime} \in \mathbb{Z} \backslash \mathbb{N} A$.

iii) $\beta, \beta^{\prime} \notin \mathbb{Z}$ but $\beta-\beta^{\prime} \in \mathbb{Z}$.

Proof. (Theorem 4.3) We have $A=(1 k a k b)$ with $1 \leq a<b, 1<k a<k b$ and $a, b$ relatively prime. From Proposition 4.11 it is enough to compute the restriction for all but finitely many $\beta \in \mathbb{C}$. We will compute the restriction of $\mathcal{M}_{A}(\beta)$ to $\left(x_{1}=0\right)$ by using an algorithm by T. Oaku and N. Takayama [30, Algorithm 5.2.8].

Let $r=k-1$ be the biggest integer root of the Bernstein polynomial $b(\tau)$ of $H_{A}(\beta)$ with respect to $\omega=(1,0,0)$ (see Corollary 4.8). Recall that $\mathcal{D}^{\prime}=\mathcal{D}_{\mathbb{C}^{2}}$ and that we are using $\left(x_{2}, x_{3}\right)$ as coordinates in $\mathbb{C}^{2}$. We consider the free $\mathcal{D}^{\prime}$-module with basis $\mathcal{B}_{k-1}:=\left\{\partial_{1}^{i}: i=0,1, \ldots, k-1\right\}$ :

$$
\left(\mathcal{D}^{\prime}\right)^{r+1}=\left(\mathcal{D}^{\prime}\right)^{k} \simeq \bigoplus_{i=0}^{k-1} \mathcal{D}^{\prime} \partial_{1}^{i} .
$$


To apply [30, Algorithm 5.2.8] to our case we will use the elements with $\omega$ order less than or equal to $k-1$, in the Groebner basis $\mathcal{G}:=\left\{P_{1}, P_{2}, P_{3}, E, R\right\}$ of $H_{A}(\beta)$. Recall that $\mathcal{G}$ is given by Lemma 4.10 for all but finitely many $\beta \in \mathbb{C}$. Each operator $\partial_{1}^{i} P_{1}, \quad \partial_{1}^{i} E, i=0, \ldots, k-1$, must be written as a $\mathbb{C}$-linear combination of monomials $x^{u} \partial^{v}$ and then substitute $x_{1}=0$ into this expression. The result is an element of $\left(\mathcal{D}^{\prime}\right)^{k}=\mathcal{D}^{\prime} \mathcal{B}_{k}$. In this case we get:

$$
\left(\partial_{1}^{i} P_{1}\right)_{\mid x_{1}=0}=P_{1} \partial_{1}^{i},\left(\partial_{1}^{i} E\right)_{\mid x_{1}=0}=\left(k a x_{2} \partial_{2}+k b x_{3} \partial_{3}-\beta+i\right) \partial_{1}^{i}, i=0, \ldots, k-1
$$

and this proves the theorem.

Remark 4.12. Let us consider $A=\left(\begin{array}{llll}1 & a_{2} & \cdots & a_{n}\end{array}\right), 1<a_{2}<\cdots<a_{n}, k=$ $\operatorname{gcd}\left(a_{n-1}, a_{n}\right)$ and $A^{\prime}=\frac{1}{k}\left(a_{n-1}, a_{n}\right)$. We can apply the Cauchy-Kovalevskaya Theorem for Gevrey series (see [20, Cor. 2.2.4]), Corollary 4.4 and [5, Prop. 4.2] to the hypergeometric system $\mathcal{M}_{A}(\beta)$ to prove that for each $\beta \in \mathbb{C}$ there exist $\beta_{i} \in \mathbb{C}$, $i=0, \ldots, k-1$, and a quasi-isomorphism

$$
\mathbb{R H o m}_{\mathcal{D}_{X}}\left(\mathcal{M}_{A}(\beta), \mathcal{O}_{\widehat{X \mid Y}}(s)\right)_{\mid X^{\prime}} \stackrel{\simeq}{\longrightarrow} \bigoplus_{i=0}^{k-1} \mathbb{R} \mathcal{H o m}_{\mathcal{D}_{X^{\prime}}}\left(\mathcal{M}_{A^{\prime}}\left(\beta_{i}\right), \mathcal{O}_{\widehat{X^{\prime} \mid Y^{\prime}}}(s)\right)
$$

for all $1 \leq s \leq \infty$, where $Y=\left(x_{n}=0\right), X^{\prime}=\left(x_{1}=x_{2}=\cdots=x_{n-2}=0\right)$ and $Y^{\prime}=Y \cap X^{\prime}$. Moreover, for all but finitely many $\beta$ we can take $\beta_{i}=\frac{\beta-i}{k}$. Notice that the coordinates in $X, Y, X^{\prime}, Y^{\prime}$ are $x=\left(x_{1}, \ldots, x_{n}\right), y=\left(x_{1}, \ldots, x_{n-1}\right)$, $x^{\prime}=\left(x_{n-1}, x_{n}\right)$ and $y^{\prime}=\left(x_{n-1}\right)$, respectively.

The last quasi-isomorphism induces a $\mathbb{C}$-linear isomorphism

$\left.\mathcal{E} t_{\mathcal{D}_{X}}^{j}\left(\mathcal{M}_{A}(\beta), \mathcal{O}_{\widehat{X \mid Y}}(s)\right)_{\left(0, \ldots, 0, \epsilon_{n-1}, 0\right)} \stackrel{\simeq}{\longrightarrow} \bigoplus_{i=0}^{k-1} \mathcal{E} x t_{\mathcal{D}_{X^{\prime}}}^{j}\left(\mathcal{M}_{A^{\prime}}\left(\beta_{i}\right), \mathcal{O}_{\widehat{X^{\prime} \mid Y^{\prime}}}(s)\right)\right)_{\left(\epsilon_{n-1}, 0\right)}$

for all $\epsilon_{n-1} \in \mathbb{C}, s \geq 1$ and $j \in \mathbb{N}$, and we also have equivalent results for $\mathcal{Q}_{Y}(s)$ and $\mathcal{Q}_{Y^{\prime}}(s)$ instead of $\mathcal{O}_{\widehat{X \mid Y}}(s)$ and $\mathcal{O}_{\widehat{X^{\prime} \mid Y^{\prime}}}(s)$.

In particular, using [7, Proposition 5.9], we have:

Proposition 4.13. Let $A=\left(\begin{array}{llll}1 & a_{2} & \cdots & a_{n}\end{array}\right)$ be an integer row matrix with $1<a_{2}<$ $\cdots<a_{n}$. Then for all $\beta \in \mathbb{C}$,

$$
\begin{aligned}
\operatorname{dim}_{\mathbb{C}}\left(\mathcal{E} x t_{\mathcal{D}_{X}}^{j}\left(\mathcal{M}_{A}(\beta), \mathcal{Q}_{Y}(s)\right)_{\left(0, \ldots, 0, \epsilon_{n-1}, 0\right)}\right) \\
\quad= \begin{cases}a_{n-1} & \text { if } s \geq a_{n} / a_{n-1}, j=0 \text { and } \epsilon_{n-1} \neq 0, \\
0 & \text { otherwise. }\end{cases}
\end{aligned}
$$

Corollary 4.14. Let $A=\left(\begin{array}{llll}1 & a_{2} & \cdots & a_{n}\end{array}\right)$ be an integer row matrix with $1<a_{2}<$ $\cdots<a_{n}$. Then for all $\beta \in \mathbb{C}$,

$$
\operatorname{Ch}\left(\operatorname{Irr}_{Y}^{(s)}\left(\mathcal{M}_{A}(\beta)\right)\right) \subseteq T_{Y}^{*} Y \cup T_{Z \cap Y}^{*} Y
$$

for $s \geq \frac{a_{n}}{a_{n-1}}$.

Proof. Here $\operatorname{Ch}\left(\operatorname{Irr}_{Y}^{(s)}\left(\mathcal{M}_{A}(\beta)\right)\right)$ is the characteristic cycle of the perverse sheaf $\operatorname{Irr}_{Y}^{(s)}\left(\mathcal{M}_{A}(\beta)\right)$ (see e.g. [19, Sec. 2.4]). The corollary follows from the inclusion

$$
\mathrm{Ch}^{(s)}\left(\mathcal{M}_{A}(\beta)\right) \subset T_{X}^{*} X \cup T_{Y}^{*} X \cup T_{Z}^{*} X
$$

for $s \geq a_{n} / a_{n-1}$ and then by applying [19, Prop. 2.4.1]. 
Proof. (Theorem 4.1) Let us consider the Whitney stratification $Y=Y_{1} \cup Y_{2}$ of $Y=\left(x_{n}=0\right) \subset \mathbb{C}^{n}$ defined as

$$
\begin{gathered}
Y_{1}:=Y \backslash(Y \cap Z) \equiv \mathbb{C}^{n-2} \times \mathbb{C}^{*}, \\
Y_{2}:=Y \cap Z \equiv \mathbb{C}^{n-2} \times\{0\} .
\end{gathered}
$$

As $\operatorname{Irr}_{Y}^{(s)}\left(\mathcal{M}_{A}(\beta)\right)$ is a perverse sheaf for all $\beta \in \mathbb{C}$ and $1 \leq s \leq \infty$ [25, Th. 6.3.3] we can apply Kashiwara's Constructibility Theorem [13, the Riemann-Hilbert correspondence (see [24] and [15, 14]) and Corollary 4.14 to prove that

$$
\mathcal{E}_{x} t_{\mathcal{D}}^{i}\left(\mathcal{M}_{A}(\beta), \mathcal{Q}_{Y}(s)\right)_{\mid Y_{j}}
$$

is a locally constant sheaf of finite rank for all $i \in \mathbb{N}, j=1,2$. To finish the proof it is enough to apply Proposition 4.13

Remark 4.15. The proof of Theorem 4.1 uses Mebkhout's result on the perversity of the irregularity, Kashiwara's Constructibility Theorem and the Riemann-Hilbert correspondence, all of them being deep results in $\mathcal{D}$-module theory. It would be interesting to give a more elementary proof of Theorem 4.1] In [7] such an elementary proof is given for the case $n=2$.

4.2. Gevrey solutions of $\mathcal{M}_{A}(\beta)$. We will compute a basis of the vector spaces $\mathcal{E} x t^{i}\left(\mathcal{M}_{A}(\beta), \mathcal{Q}_{Y}(s)\right)_{p}$ for all $p \in Y, 1 \leq s \leq \infty, \beta \in \mathbb{N}, i \in \mathbb{N}$ and $A=\left(\begin{array}{llll}1 & a_{2} & \cdots & a_{n}\end{array}\right)$ is an integer row matrix with $1<a_{2}<\cdots<a_{n}$. In fact it is enough to achieve the computation for $i=0$ and $p \in Y \backslash Z$; otherwise the corresponding germ is zero by Theorem 4.1.

Lemma 4.16. Let $A=\left(\begin{array}{llll}1 & a_{2} & \cdots & a_{n}\end{array}\right)$ be an integer row matrix with $1<a_{2}<\cdots<$ $a_{n}$ and $\omega \in \mathbb{R}_{>0}^{n}$ satisfying

a) $w_{i}>a_{i} \omega_{1}$ for $2 \leq i \leq n-2$ or $i=n$,

b) $a_{n-1} \omega_{1}>\omega_{n-1}$,

c) $\omega_{n-1}>\omega_{1}, \ldots, \omega_{n-2}$.

Then $H_{A}(\beta)$ has $a_{n-1}$ exponents with respect to $\omega$, and they have the form

$$
v^{j}=\left(j, 0, \ldots, 0, \frac{\beta-j}{a_{n-1}}, 0\right) \in \mathbb{C}^{n},
$$

$j=0,1, \ldots, a_{n-1}-1$.

Proof. The notion of exponent is given in [30, page 92]. The toric ideal $I_{A}$ is generated by $P_{1, i}=\partial_{1}^{a_{i}}-\partial_{i} \in \mathbb{C}[\partial], i=2, \ldots, n$.

Let $\omega=\left(\omega_{1}, \ldots, \omega_{n}\right) \in \mathbb{R}_{>0}^{n}$ be a weight vector satisfying the statement of the lemma. We have:

$$
\operatorname{in}_{(-\omega, \omega)} P_{1, i}= \begin{cases}\partial_{i} & \text { if } i=2, \ldots, n-2, n, \\ \partial_{1}^{a_{n-1}} & \text { if } i=n-1 .\end{cases}
$$

In particular $\left\{P_{1, i}: \quad i=2, \ldots, n\right\}$ is a Groebner basis of $I_{A}$ with respect to $(-\omega, \omega)$ and then

$$
\operatorname{in}_{\omega} I_{A}=\left\langle\partial_{2}, \ldots, \partial_{n-2}, \partial_{1}^{a_{n-1}}, \partial_{n}\right\rangle .
$$

The standard pairs of $\operatorname{in}_{\omega}\left(I_{A}\right)$ are ([30, Sec. 3.2]):

$$
\mathcal{S}\left(\operatorname{in}_{\omega}\left(I_{A}\right)\right)=\left\{\left(\partial_{1}^{j},\{n-1\}\right): j=0,1, \ldots, a_{n-1}-1\right\} .
$$


To the standard pair $\left(\partial_{1}^{j},\{n-1\}\right)$ we associate, following [30, page 108], the fake exponent

$$
v^{j}=\left(j, 0, \ldots, 0, \frac{\beta-j}{a_{n-1}}, 0\right)
$$

of the module $\mathcal{M}_{A}(\beta)$ with respect to $\omega$. It is easy to prove that these fake exponents are in fact exponents since they have minimal negative support [30, Th. 3.4.13].

Remark 4.17. With the above notation, the $\Gamma$-series $\phi_{v^{j}}$ associated with $v^{j}$ for $j=0, \ldots, a_{n-1}-1$ is defined as

$$
\phi_{v^{j}}=x^{v^{j}} \sum_{u \in L_{A}} \Gamma\left[v^{j} ; u\right] x^{u},
$$

where $L_{A}=\operatorname{ker}_{\mathbb{Z}}(A)$ is the lattice generated by the vectors $\left\{u^{2}, \ldots, u^{n}\right\}$ and $u^{i}$ is the $(i-1)$-th row of the matrix

$$
\left(\begin{array}{rccccccc}
-a_{2} & 1 & 0 & \cdots & 0 & 0 & 0 & 0 \\
\vdots & \vdots & \vdots & \vdots & \vdots & \vdots & \vdots & \vdots \\
-a_{n-2} & 0 & 0 & \cdots & 0 & 1 & 0 & 0 \\
a_{n-1} & 0 & 0 & \cdots & 0 & 0 & -1 & 0 \\
-a_{n} & 0 & 0 & \cdots & 0 & 0 & 0 & 1
\end{array}\right) .
$$

For any $\mathbf{m}=\left(m_{2}, \ldots, m_{n}\right) \in \mathbb{Z}^{n-1}$, let $u(\mathbf{m}):=\sum_{i=2}^{n} m_{i} u^{i} \in L_{A}$. We can write

$$
\phi_{v^{j}}=x^{v^{j}} \sum_{\substack{m_{2}, \ldots, m_{n-1}, m_{n} \geq 0 \\ \sum_{i \neq n-1} a_{i} m_{i} \leq j+a_{n-1} m_{n-1}}} \Gamma\left[v^{j} ; u(\mathbf{m})\right] x^{u(\mathbf{m})}
$$

for $j=0,1, \ldots, a_{n-1}-1$. We have for $\mathbf{m}=\left(m_{2}, \ldots, m_{n}\right) \in \mathbb{N}^{n-1}$ such that $j-\sum_{i \neq n-1} a_{i} m_{i}+a_{n-1} m_{n-1} \geq 0$,

$$
\Gamma\left[v^{j} ; u(\mathbf{m})\right]=\frac{\left(\frac{\beta-j}{a_{n-1}}\right)_{m_{n-1}} j !}{m_{2} ! \cdots m_{n-2} ! m_{n} !\left(j-\sum_{i \neq n-1} a_{i} m_{i}+a_{n-1} m_{n-1}\right) !}
$$

and

$$
x^{u(\mathbf{m})}=x_{1}^{-\sum_{i \neq n-1} a_{i} m_{i}+a_{n-1} m_{n-1}} x_{2}^{m_{2}} \cdots x_{n-2}^{m_{n-2}} x_{n-1}^{-m_{n-1}} x_{n}^{m_{n}} .
$$

Proposition 4.18. Let $A=\left(1 a_{2} \cdots a_{n}\right)$ be an integer row matrix with $1<a_{2}<$ $\cdots<a_{n}, Y=\left(x_{n}=0\right) \subset X$ and $Z=\left(x_{n-1}=0\right) \subset X$. Then we have:

$$
\mathcal{E} x t^{0}\left(\mathcal{M}_{A}(\beta), \mathcal{O}_{\widehat{X \mid Y}}\right)_{p}=\sum_{j=0}^{a_{n-1}-1} \mathbb{C} \phi_{v^{j}, p}
$$

for all $\beta \in \mathbb{C}, p \in Y \backslash Z$.

Proof. Step 1. Using [10] and [30] we will describe $a_{n-1}$ linearly independent solutions of $\mathcal{M}_{A}(\beta)_{p}$, living in some Nilsson series ring. Then, using initial ideals, we will prove that an upper bound of the dimension of $\mathcal{E} x t^{0}\left(\mathcal{M}_{A}(\beta), \mathcal{O}_{\widehat{X \mid Y}}\right)_{p}$ is $a_{n-1}$ for $p$ in $Y \backslash Z$.

First of all, the series

$$
\left\{\phi_{v^{j}} \mid j=0, \ldots, a_{n-1}-1\right\} \subset x^{v^{j}} \mathbb{C}\left[\left[x_{1}^{ \pm 1}, x_{2}, \ldots, x_{n-2}, x_{n-1}^{-1}, x_{n}\right]\right],
$$


described in Remark 4.17, are linearly independent since $\operatorname{in}_{\omega}\left(\phi_{v^{j}}\right)=x^{v^{j}}$ for $0 \leq$ $j \leq a_{n-1}-1$. They are solutions of the system $\mathcal{M}_{A}(\beta)$ (see [8, [10, Sec. 1], [30, Sec. 3.4]).

On the other hand

$$
\operatorname{dim}_{\mathbb{C}} \mathcal{E} x t^{0}\left(\mathcal{M}_{A}(\beta), \mathcal{O}_{\widehat{X \mid Y}}\right)_{p} \leq a_{n-1}
$$

for $p=\left(\epsilon_{1}, \ldots, \epsilon_{n-1}, 0\right), \epsilon_{n-1} \neq 0$. This follows from the following facts:

a) The initial ideal $\operatorname{in}_{\omega}\left(I_{A}\right)$ equals $\left\langle\partial_{2}, \ldots, \partial_{n-2}, \partial_{1}^{a_{n-1}}, \partial_{n}\right\rangle$ for $\omega$ as in Lemma 4.16,

b) The germ of $E$ at $p$ is nothing but $E_{p}:=E+\sum_{i=1}^{n-1} a_{i} \epsilon_{i} \partial_{i}$ (here $a_{1}=1$ ) and satisfies

$$
\operatorname{in}_{(-\omega, \omega)}\left(E_{p}\right)=a_{n-1} \epsilon_{n-1} \partial_{n-1} .
$$

c) By [30, Th. 2.5.5], if $f \in \mathcal{O}_{\widehat{X \mid Y}, p}$ is a solution of the ideal $H_{A}(\beta)$, then $\operatorname{in}_{\omega}(f)$ must be annihilated by $\operatorname{in}_{(-\omega, \omega)}\left(H_{A}(\beta)\right)$. That proves inequality (4.1).

Step 2. It is easy to prove, using standard estimates, that the series $\phi_{v^{j}, p}$ are in fact in $\mathcal{O}_{\widehat{X \mid Y}, p}$ for all $p \in Y \backslash Z$. Then by step 1 , they form a basis of the vector space $\mathcal{E} x t^{0}\left(\mathcal{M}_{A}(\beta), \mathcal{O}_{\widehat{X \mid Y}}\right)_{p}$ for $p \in Y \backslash Z$.

Remark 4.19. We will prove (see Theorem 4.21) that the Gevrey index of $\phi_{v^{j}, p}$ is $a_{n} / a_{n-1}$ for $\beta \in \mathbb{C}$ and $p \in Y \backslash Z$ except for $\beta \in \mathbb{N}$ and $j=q$ the unique integer $0 \leq q \leq a_{n-1}-1$ such that $\beta-q \in a_{n-1} \mathbb{N}$. In that case, $\phi_{v^{q}}$ is a polynomial (see details in Remark 4.22).

Proposition 4.20. Let $A=\left(\begin{array}{llll}1 & a_{2} & \cdots & a_{n}\end{array}\right)$ be an integer row matrix with $1<a_{2}<$ $\cdots<a_{n}, Y=\left(x_{n}=0\right) \subset X$. Then we have:

i) For each $\beta \in \mathbb{C} \backslash \mathbb{N}$ we have $\mathcal{E} x t^{j}\left(\mathcal{M}_{A}(\beta), \mathcal{O}_{X \mid Y}\right)=0$ for all $j \in \mathbb{N}$.

ii) For each $\beta \in \mathbb{N}$ the sheaf $\mathcal{E} x t^{j}\left(\mathcal{M}_{A}(\beta), \mathcal{O}_{X \mid Y}\right)$ is locally constant on $Y$ of rank 1 for $j=0,1$ and it is zero for $j \geq 2$.

Proof. For all $\beta \in \mathbb{C}$ the characteristic variety of $\mathcal{M}_{A}(\beta)$ is $\operatorname{Ch}\left(\mathcal{M}_{A}(\beta)\right)=T_{X}^{*} X \cup$ $T_{Y}^{*} X$ (see e.g. [1]). Then from Kashiwara's Constructibility Theorem [13] we have that, for all $j \in \mathbb{N}$, the sheaf

$$
\mathcal{E} x t^{j}\left(\mathcal{M}_{A}(\beta), \mathcal{O}_{X}\right)_{\mid Y}=\mathcal{E} x t^{j}\left(\mathcal{M}_{A}(\beta), \mathcal{O}_{X \mid Y}\right)_{\mid Y}
$$

is locally constant.

Assume $\beta \notin \mathbb{N}$. From Corollary 4.4 and Proposition 4.11 we have that there exists $m \in \mathbb{N}$ such that $\mathcal{M}_{A}(\beta) \simeq \mathcal{M}_{A}(\beta-m)$ and

$$
\mathcal{M}_{A}(\beta)_{\mid X^{\prime}} \simeq \mathcal{M}_{A}(\beta-m)_{\mid X^{\prime}} \simeq \bigoplus_{i=0}^{k-1} \mathcal{M}_{\left(a_{n-1}^{\prime} a_{n}^{\prime}\right)}\left(\frac{\beta-m-i}{k}\right)
$$

with $X^{\prime}=\left(x_{1}=\cdots=x_{n-2}=0\right) \subset X, k=\operatorname{gcd}\left(a_{n-1}, a_{n}\right)$ and $a_{\ell}^{\prime}=\frac{a_{\ell}}{k}$ for $\ell=n-1, n$. Then by applying the Cauchy-Kovalevskaya Theorem (see Remark 4.12) we get:

$$
\mathcal{E} t^{j}\left(\mathcal{M}_{A}(\beta), \mathcal{O}_{X \mid Y}\right)_{\mid X^{\prime}} \simeq \bigoplus_{i=0}^{k-1} \mathcal{E} x t^{j}\left(\mathcal{M}_{\left(a_{n-1}^{\prime} a_{n}^{\prime}\right)}\left(\frac{\beta-m-i}{k}\right), \mathcal{O}_{X^{\prime} \mid Y^{\prime}}\right)
$$

with $Y^{\prime}=X^{\prime} \cap Y$. 
As $\beta \notin \mathbb{N}$, then $\frac{\beta-m-i}{k} \notin a_{n-1}^{\prime} \mathbb{N}+a_{n}^{\prime} \mathbb{N}$ for $i=0, \ldots, k-1$. Then part $\left.i\right)$ follows from [7, Proposition 4.5].

Now assume that $\beta \in \mathbb{N}$. From Corollary 4.4 and Proposition 4.11 we have that there exists $m \in \mathbb{N}$ such that $\mathcal{M}_{A}(\beta) \simeq \mathcal{M}_{A}(\beta+m)$ and

$$
\mathcal{M}_{A}(\beta)_{\mid X^{\prime}} \simeq \mathcal{M}_{A}(\beta+m)_{\mid X^{\prime}} \simeq \bigoplus_{i=0}^{k-1} \mathcal{M}_{\left(a_{n-1}^{\prime} a_{n}^{\prime}\right)}\left(\frac{\beta+m-i}{k}\right) .
$$

Then by applying the Cauchy-Kovalevskaya Theorem (see Remark 4.12) we get:

$$
\mathcal{E}_{x} t^{j}\left(\mathcal{M}_{A}(\beta), \mathcal{O}_{X \mid Y}\right)_{\mid X^{\prime}} \simeq \bigoplus_{i=0}^{k-1} \mathcal{E} x t^{j}\left(\mathcal{M}_{\left(a_{n-1}^{\prime} a_{n}^{\prime}\right)}\left(\frac{\beta+m-i}{k}\right), \mathcal{O}_{X^{\prime} \mid Y^{\prime}}\right)
$$

By [7] this last module is in fact equal to

$$
\mathcal{E} x t^{j}\left(\mathcal{M}_{\left(a_{n-1}^{\prime} a_{n}^{\prime}\right)}\left(\frac{\beta+m-i_{0}}{k}\right), \mathcal{O}_{X^{\prime} \mid Y^{\prime}}\right)
$$

where $i_{0}$ is the unique integer number such that $0 \leq i_{0} \leq k-1$ and $\beta+m-i_{0} \in k \mathbb{N}$. Then part ii) follows from [7, Proposition 4.6].

Theorem 4.21. Let $A=\left(\begin{array}{llll}1 & a_{2} & \cdots & a_{n}\end{array}\right)$ be an integer row matrix with $1<a_{2}<$ $\cdots<a_{n}, Y=\left(x_{n}=0\right) \subset X$ and $Z=\left(x_{n-1}=0\right) \subset X$. Then we have:

i)

$$
\mathcal{E}_{x} t^{0}\left(\mathcal{M}_{A}(\beta), \mathcal{O}_{\widehat{X \mid Y}}(s)\right)_{p}=\bigoplus_{j=0}^{a_{n-1}-1} \mathbb{C} \phi_{v^{j}, p}
$$

ii)

for all $\beta \in \mathbb{C}, p \in Y \backslash Z$ and $s \geq a_{n} / a_{n-1}$.

$$
\mathcal{E} x t^{0}\left(\mathcal{M}_{A}(\beta), \mathcal{O}_{\widehat{X \mid Y}}(s)\right)_{p}=\left\{\begin{array}{cc}
0 & \text { if } \beta \notin \mathbb{N} \\
\mathbb{C} \phi_{v^{q}} & \text { if } \beta \in \mathbb{N}
\end{array}\right.
$$

for all $p \in Y \backslash Z$ and $1 \leq s<a_{n} / a_{n-1}$, where $q$ is the unique element in $\left\{0,1, \ldots, a_{n-1}-1\right\}$ satisfying $\frac{\beta-q}{a_{n-1}} \in \mathbb{N}$ and $\phi_{v^{q}}$ is a polynomial.

Proof. i) Let us consider $a_{n} / a_{n-1} \leq s \leq \infty$ and $p \in Y \backslash Z$. Assume first that $\beta \notin \mathbb{N}$. By Proposition 4.20 and the long exact sequence of cohomology associated with the short exact sequence (1.1) we have that

$$
\mathcal{E}_{x} t^{0}\left(\mathcal{M}_{A}(\beta), \mathcal{O}_{\widehat{X \mid Y}}(s)\right)_{p} \simeq \mathcal{E} x t^{0}\left(\mathcal{M}_{A}(\beta), \mathcal{Q}_{Y}(s)\right)_{p}
$$

and by Theorem 4.1 this last vector space has dimension $a_{n-1}$. As

$$
\mathcal{E}_{x} t^{0}\left(\mathcal{M}_{A}(\beta), \mathcal{O}_{\widehat{X \mid Y}}(s)\right)_{p} \subset \mathcal{E} x t^{0}\left(\mathcal{M}_{A}(\beta), \mathcal{O}_{\widehat{X \mid Y}}\right)_{p},
$$

part i) follows from Proposition 4.18 if $\beta \notin \mathbb{N}$.

Now assume that $\beta \in \mathbb{N}$. Applying the long exact sequence of cohomology associated with the short exact sequence (1.1), Theorem 4.1 and Proposition 4.20 we get an exact sequence of vector spaces

$$
0 \rightarrow \mathbb{C} \rightarrow \mathcal{L}_{1} \rightarrow \mathcal{E} x t^{0}\left(\mathcal{M}_{A}(\beta), \mathcal{Q}_{Y}(s)\right)_{p} \rightarrow \mathbb{C} \rightarrow \mathcal{L}_{2} \rightarrow 0
$$

where $\mathcal{L}_{1}=\mathcal{E} x t^{0}\left(\mathcal{M}_{A}(\beta), \mathcal{O}_{\widehat{X \mid Y}}(s)\right)_{p}$ and $\mathcal{L}_{2}=\mathcal{E} x t^{1}\left(\mathcal{M}_{A}(\beta), \mathcal{O}_{\widehat{X \mid Y}}(s)\right)_{p}$. Let us write $\nu_{i}=\operatorname{dim}_{\mathbb{C}}\left(\mathcal{L}_{i}\right)$. By Theorem 4.1 we also have $\nu_{1}=a_{n-1}+\nu_{2}$. On the other 
hand, by Proposition 4.18 we know that $\nu_{1} \leq a_{n-1}$. This implies that $\nu_{1}=a_{n-1}$ and $\mathcal{L}_{2}=\{0\}$. In particular we have the equality

$$
\mathcal{E} x t^{0}\left(\mathcal{M}_{A}(\beta), \mathcal{O}_{\widehat{X \mid Y}}(s)\right)_{p} \subset \mathcal{E} x t^{0}\left(\mathcal{M}_{A}(\beta), \mathcal{O}_{\widehat{X \mid Y}}\right)_{p}
$$

Part i) also follows from Proposition 4.18 if $\beta \in \mathbb{N}$.

Let us prove part ii). First of all, by Theorem 4.1, $\mathcal{E} x t^{j}\left(\mathcal{M}_{A}(\beta), \mathcal{Q}_{Y}(s)\right)_{p}=0$ for all $j \in \mathbb{N}$. Then the result follows from the long exact sequence of cohomology associated with the short exact sequence (1.1) and Proposition 4.20.

Remark 4.22. Let us recall here the notation introduced in Lemma 4.16. For $A=$ $\left(1 a_{2} \cdots a_{n}\right)$ an integer row matrix with $1<a_{2}<\cdots<a_{n}$ and $\omega \in \mathbb{R}_{>0}^{n}$ satisfying

(1) $w_{i}>a_{i} \omega_{1}$, for $2 \leq i \leq n-2$ or $i=n$,

(2) $a_{n-1} \omega_{1}>\omega_{n-1}$,

(3) $\omega_{n-1}>\omega_{1}, \ldots, \omega_{n-2}$,

we have proved (see Lemma 4.16) that $H_{A}(\beta)$ has $a_{n-1}$ exponents with respect to $\omega$ and that they have the form:

$$
v^{j}=\left(j, 0, \ldots, 0, \frac{\beta-j}{a_{n-1}}, 0\right) \in \mathbb{C}^{n},
$$

$j=0,1, \ldots, a_{n-1}-1$.

The corresponding $\Gamma$-series $\phi_{v^{j}}$ is defined as:

$$
\phi_{v^{j}}=x^{v^{j}} \sum_{\substack{m_{2}, \ldots, m_{n-1}, m_{n} \geq 0 \\ \sum_{i \neq n-1} a_{i} m_{i} \leq j+a_{n-1} m_{n-1}}} \Gamma\left[v^{j} ; u(\mathbf{m})\right] x^{u(\mathbf{m})}
$$

for $j=0,1, \ldots, a_{n-1}-1$, where for any $\mathbf{m}=\left(m_{2}, \ldots, m_{n}\right) \in \mathbb{Z}^{n-1}$ we let $u(\mathbf{m}):=$ $\sum_{i=2}^{n} m_{i} u^{i} \in L_{A}$.

For $\mathbf{m}=\left(m_{2}, \ldots, m_{n}\right) \in \mathbb{N}^{n-1}$ such that $j-\sum_{i \neq n-1} a_{i} m_{i}+a_{n-1} m_{n-1} \geq 0$, we have

$$
\Gamma\left[v^{j} ; u(\mathbf{m})\right]=\frac{\left(\frac{\beta-j}{a_{n-1}}\right)_{m_{n-1}} j !}{m_{2} ! \cdots m_{n-2} ! m_{n} !\left(j-\sum_{i \neq n-1} a_{i} m_{i}+a_{n-1} m_{n-1}\right) !}
$$

and

$$
x^{u(\mathbf{m})}=x_{1}^{-\sum_{i \neq n-1} a_{i} m_{i}+a_{n-1} m_{n-1}} x_{2}^{m_{2}} \cdots x_{n-2}^{m_{n-2}} x_{n-1}^{-m_{n-1}} x_{n}^{m_{n}} .
$$

If $\beta \in \mathbb{N}$, then there exists a unique $0 \leq q \leq a_{n-1}-1$ such that $\beta-q \in a_{n-1} \mathbb{N}$. Let us write $m_{0}=\frac{\beta-q}{a_{n-1}}$.

Then for $m \in \mathbb{N}$ big enough, $m_{0}-a_{n} m$ is a negative integer and the coefficient $\Gamma\left[v^{q} ; u(\mathbf{m})\right]$ is zero and then $\phi_{v^{q}}$ is a polynomial in $\mathbb{C}[x]$.

Recall that $u^{n-1}=\left(a_{n-1}, 0, \ldots,-1,0\right) \in L_{A}$ and let us write

$$
\begin{aligned}
\widetilde{v^{q}}=v^{q}+\left(m_{0}+1\right) u^{n-1} & =\left(q+\left(m_{0}+1\right) a_{n-1}, 0, \ldots, 0,-1,0\right) \\
& =\left(\beta+a_{n-1}, 0, \ldots, 0,-1,0\right) .
\end{aligned}
$$

We have $A \widetilde{v^{q}}=\beta$ and the corresponding $\Gamma$-series is

$$
\phi_{\widetilde{v^{q}}}=x^{\widetilde{v^{q}}} \sum_{\mathbf{m} \in M(q)} \Gamma\left[\widetilde{v^{q}} ; u(\mathbf{m})\right] x^{u(\mathbf{m})}
$$

where for $\mathbf{m}=\left(m_{2}, \ldots, m_{n}\right) \in \mathbb{Z}^{n}$ one has $u(\mathbf{m})=\sum_{i=2}^{n} m_{i} u^{i}$ and

$$
M(q):=\left\{\left(m_{2}, \ldots, m_{n}\right) \in \mathbb{N}^{n-1} \mid q+\left(m_{0}+m_{n-1}+1\right) a_{n-1}-\sum_{i \neq n-1} a_{i} m_{i} \geq 0\right\} .
$$


Let us notice that $\widetilde{v^{q}}$ does not have minimal negative support (see 30, pp. 132133]), and then the $\Gamma$-series $\phi_{\widetilde{v^{q}}}$ is not a solution of $H_{A}(\beta)$. We will prove in Theorem 4.23 that $H_{A}(\beta)_{p}\left(\phi_{\widetilde{v}^{q}, p}\right) \subset \mathcal{O}_{X, p}$ for all $p \in Y \backslash Z$ and that $\phi_{\widetilde{v^{q}}, p}$ is a Gevrey series of index $a_{n} / a_{n-1}$.

The second main result of this section is the following.

Theorem 4.23. Let $A=\left(\begin{array}{llll}1 & a_{2} & \cdots & a_{n}\end{array}\right)$ be an integer row matrix with $1<a_{2}<$ $\cdots<a_{n}, Y=\left(x_{n}=0\right) \subset X$ and $Z=\left(x_{n-1}=0\right) \subset X$. Then for all $p \in Y \backslash Z$ and $s \geq a_{n} / a_{n-1}$ we have:

i) If $\beta \notin \mathbb{N}$, then:

$$
\mathcal{E}_{x} t^{0}\left(\mathcal{M}_{A}(\beta), \mathcal{Q}_{Y}(s)\right)_{p}=\bigoplus_{j=0}^{a_{n-1}-1} \mathbb{C} \overline{\phi_{v^{j}, p}} .
$$

ii) If $\beta \in \mathbb{N}$, then there exists a unique $q \in\left\{0, \ldots, a_{n-1}-1\right\}$ such that $\frac{\beta-q}{a_{n-1}} \in \mathbb{N}$ and we have:

$$
\mathcal{E} x t^{0}\left(\mathcal{M}_{A}(\beta), \mathcal{Q}_{Y}(s)\right)_{p}=\bigoplus_{q \neq j=0}^{a_{n-1}-1} \mathbb{C} \overline{\phi_{v^{j}, p}} \oplus \mathbb{C} \overline{\phi_{\widetilde{v^{q}}, p}} .
$$

Here $\bar{\phi}$ stands for the class modulo $\mathcal{O}_{X \mid Y, p}$ of $\phi \in \mathcal{O}_{\widehat{X \mid Y}, p}(s)$.

Proof. Part i) follows from Theorem 4.21 and Proposition 4.20 using the long exact sequence of cohomology.

Let us prove ${ }^{i i}$ ). Since $\mathcal{E} x t^{1}\left(\mathcal{M}_{A}(\beta), \mathcal{Q}_{Y}(s)\right)=0$ (see Theorem4.1) and applying Theorem 4.21, Proposition 4.20 and the long exact sequence in cohomology we get that

$$
\mathcal{E}^{1} t^{1}\left(\mathcal{M}_{A}(\beta), \mathcal{O}_{\widehat{X \mid Y}}(s)\right)_{\mid Y \backslash Z}
$$

is zero for $s \geq a_{n} / a_{n-1}$ and locally constant of rank 1 for $1 \leq s<a_{n} / a_{n-1}$. We also have that

$$
\mathcal{E} t^{1}\left(\mathcal{M}_{A}(\beta), \mathcal{O}_{\widehat{X \mid Y}}(s)\right)_{\mid Y \cap Z}
$$

is locally constant of rank 1 for all $s \geq 1$.

Assume $s \geq a_{n} / a_{n-1}$. We consider the following long exact sequence associated with the short exact sequence (1.1) (with $p \in Y \backslash Z$ and $\mathcal{M}=\mathcal{M}_{A}(\beta)$ )

$$
\begin{aligned}
0 \rightarrow & \mathcal{E} x t^{0}\left(\mathcal{M}, \mathcal{O}_{X \mid Y}\right)_{p} \rightarrow \mathcal{E} x t^{0}\left(\mathcal{M}, \mathcal{O}_{\widehat{X \mid Y}}(s)\right)_{p} \stackrel{\rho}{\rightarrow} \\
& \mathcal{E} x t^{0}\left(\mathcal{M}, \mathcal{Q}_{Y}(s)\right)_{p} \rightarrow \mathcal{E} x t^{1}\left(\mathcal{M}, \mathcal{O}_{X \mid Y}\right)_{p} \rightarrow 0
\end{aligned}
$$

We also have

$$
\begin{gathered}
\mathcal{E} x t^{0}\left(\mathcal{M}_{A}(\beta), \mathcal{O}_{X \mid Y}\right)_{p} \simeq \mathbb{C} \\
\mathcal{E} x t^{0}\left(\mathcal{M}_{A}(\beta), \mathcal{O}_{\widehat{X \mid Y}}(s)\right)_{p} \simeq \mathbb{C}^{a_{n-1}}, \\
\mathcal{E} x t^{0}\left(\mathcal{M}_{A}(\beta), \mathcal{Q}_{Y}(s)\right)_{p} \simeq \mathbb{C}^{a_{n-1}}, \\
\mathcal{E}_{x t^{1}\left(\mathcal{M}_{A}(\beta), \mathcal{O}_{X \mid Y}\right)_{p}} \simeq \mathbb{C} .
\end{gathered}
$$

Since $\beta \in \mathbb{N}$ there exists a unique $q=0,1, \ldots, a_{n-1}-1$ such that $\frac{\beta-q}{a_{n-1}} \in \mathbb{N}$ and then $\phi_{v^{q}} \in \mathbb{C}[x]$ generates $\mathcal{E} x t^{0}\left(\mathcal{M}_{A}(\beta), \mathcal{O}_{X \mid Y}\right)_{p}=\operatorname{Ker}(\rho)$. 
Using the exact sequence (4.3) and the first isomorphism theorem we get that the family

$$
\left\{\overline{\phi_{v^{j}, p}}: 0 \leq j \leq a_{n-1}-1, j \neq q\right\}
$$

is linearly independent in $\mathcal{Q}_{Y}(s)_{p}$ for all $p \in Y \backslash Z$.

In a similar way to the proof of Theorem 4.1 it can be proved that $\phi_{\widetilde{v^{q}}, p} \in$ $\mathcal{O}_{\widehat{X \mid Y}}(s)_{p}$ for all $p \in Y \backslash Z$ and $s \geq a_{n} / a_{n-1}$.

Writing $t_{n-1}=x_{n-1}^{-1}$ and defining

$$
\psi_{\widetilde{v^{q}}}\left(x_{1}, \ldots, x_{n-2}, t_{n-1}, x_{n}\right):=x^{-\widetilde{v^{q}}} \phi_{\widetilde{v^{q}}}\left(x_{1}, \ldots, x_{n-2}, \frac{1}{t_{n-1}}, x_{n}\right),
$$

we have that

$$
\psi_{\widetilde{v^{q}}} \in \mathbb{C}\left[\left[x_{1}, \ldots, x_{n-2}, t_{n-1}, x_{n}\right]\right] .
$$

Taking the subsum of $\psi_{\widetilde{v^{q}}}$ for $m_{2}=\cdots=m_{n-2}=0, m_{n}=a_{n-1} m, m_{n-1}=$ $a_{n} m, m \in \mathbb{N}$, we get the power series

$$
\sum_{m \geq 0} c_{m}\left(t_{n-1}^{a_{n}} x_{n}^{a_{n-1}}\right)^{m}
$$

where

$$
c_{m}=\frac{(-1)^{a_{n} m}\left(a_{n} m\right) !}{\left(a_{n-1} m\right) !} .
$$

This power series has Gevrey index $a_{n} / a_{n-1}$ with respect to $x_{n}=0$. Then $\phi_{\widetilde{v^{q}}}$ has Gevrey index $a_{n} / a_{n-1}$.

We have $E\left(\phi_{\widetilde{v^{q}}}\right)=P_{i}\left(\phi_{\widetilde{v^{q}}}\right)=0$, for all $i=1,2, \ldots, n-2, n$ and $P_{n-1}\left(\phi_{\widetilde{v^{q}}}\right)$ is a meromorphic function with poles along $Z$ (and holomorphic on $X \backslash Z$ ):

$P_{n-1}\left(\phi_{\widetilde{v^{q}}}\right)=\sum_{\underline{m} \in \widetilde{M}(q)} \frac{\left(\beta+a_{n-1}\right) ! x_{1}^{q-\sum_{i \neq n-1} a_{i} m_{i}+a_{n-1}\left(m_{0}+1\right)} x_{2}^{m_{2}} \cdots x_{n-2}^{m_{n-2}} x_{n-1}^{-1} x_{n}^{m_{n}}}{m_{2} ! \cdots m_{n-2} ! m_{n} !\left(q-\sum_{i \neq n-1} a_{i} m_{i}+a_{n-1}\left(m_{0}+1\right)\right) !}$,

where

$\widetilde{M}(q)=\left\{\left(m_{2}, \ldots, m_{n-2}, m_{n}\right) \in \mathbb{N}^{n-2} \mid \sum a_{i} m_{i} \leq q+a_{n-1}\left(m_{0}+1\right)=\beta+a_{n-1}\right\}$

is a finite set (recall that $\left.m_{0}=\frac{\beta-q}{a_{n-1}} \in \mathbb{N}\right)$.

In particular, $H_{A}(\beta)\left(\phi_{\widetilde{v^{q}}}\right) \subseteq \mathcal{O}_{X}(X \backslash Z)$.

So,

$$
\overline{\phi_{\widetilde{v^{q}}, p}} \in \mathcal{E} x t^{0}\left(\mathcal{M}_{A}(\beta), \mathcal{Q}_{Y}(s)\right)_{p}
$$

for all $p \in Y \backslash Z$ and $s \geq a_{n} / a_{n-1}$.

In order to finish the proof we will see that for all $\lambda_{j} \in \mathbb{C}\left(j=0, \ldots, a_{n-1}-1\right.$; $j \neq q$ ) and for all $p \in Y \backslash Z$ we have

$$
\phi_{\widetilde{v^{q}, p}}-\sum_{j \neq q} \lambda_{j} \phi_{v^{j}, p} \notin \mathcal{O}_{X \mid Y, p}
$$

Let us write

$$
\psi_{v^{j}}\left(x_{1}, \ldots, x_{n-2}, t_{n-1}, x_{n}\right):=\phi_{v^{j}}\left(x_{1}, \ldots, x_{n-2}, \frac{1}{t_{n-1}}, x_{n}\right) .
$$

Assume to the contrary that there exist $p \in Y \backslash Z$ and $\lambda_{j} \in \mathbb{C}$ such that:

$$
\phi_{\widetilde{v^{q}, p}}-\sum_{j \neq q} \lambda_{j} \phi_{v^{j}, p} \in \mathcal{O}_{X \mid Y, p}
$$


Let us consider the holomorphic function at $p$ defined as

$$
f:=x^{\widetilde{v^{q}}} \psi_{\widetilde{v^{q}, p}}-\sum_{j \neq q} \lambda_{j} \psi_{v^{j}, p}
$$

We have the following equality of holomorphic functions at $p$ :

$$
\rho_{s}\left(f+\sum_{j \neq q} \lambda_{j} \psi_{v^{j}}\right)=\rho_{s}\left(x^{\widetilde{v^{q}}} \psi_{\widetilde{v^{q}}}\right)
$$

for $s>a_{n}$.

The function $\rho_{s}\left(x^{\widetilde{v^{q}}} \psi_{\widetilde{v^{q}}}\right)$ is holomorphic in $\mathbb{C}^{n}$ while each $\rho_{s}\left(\psi_{v^{j}}\right)$ has the form $t_{n-1}^{-\frac{\beta-j}{a_{n-1}}} \psi_{j}$ with $\psi_{j}$ holomorphic in $\mathbb{C}^{n}$.

Making a loop around the $t_{n-1}$-axis $\left(\log t_{n-1} \mapsto \log t_{n-1}+2 \pi i\right)$ we get the equality:

$$
\rho_{s}\left(\widehat{f}+\sum_{j \neq q} c_{j} \lambda_{j} \psi_{v^{j}}\right)=\rho_{s}\left(x^{\widetilde{v^{q}}} \psi_{\widetilde{v^{q}}}\right),
$$

where $c_{j}=e^{-\frac{\beta-j}{a_{n-1}} 2 \pi i} \neq 1$ (since $\frac{\beta-j}{a_{n-1}} \notin \mathbb{Z}$ for all $j \neq q$ ) and $\widehat{f}$ is obtained from $f$ after the loop. Since $f$ is holomorphic at $p$, then $\widehat{f}$ is also. Subtracting both equalities we get:

$$
\rho_{s}\left(\widehat{f}-f+\sum_{j \neq q}\left(c_{j}-1\right) \lambda_{j} \psi_{v^{j}}\right)=0
$$

and then

$$
\sum_{j \neq q}\left(c_{j}-1\right) \lambda_{j} \psi_{v^{j}}=f-\widehat{f}
$$

in the neighborhood of $p$. This contradicts the fact that the power series $\left\{\phi_{v^{j}}\right.$ : $\left.j \neq q, 0 \leq j \leq a_{n-1}-1\right\}$ are linearly independent modulo $\mathcal{O}_{X \mid Y, p}$ (here we have $\left.c_{j}-1 \neq 0\right)$. This proves the theorem.

Corollary 4.24. If $\beta \in \mathbb{N}$, then for all $p \in Y \backslash Z$ the vector space $\mathcal{E} x t^{1}\left(\mathcal{M}_{A}(\beta)\right.$, $\left.\mathcal{O}_{X \mid Y}\right)_{p}$ is generated by the class of:

$$
\begin{gathered}
\left(P_{2}\left(\phi_{\widetilde{v}^{q}}\right), \ldots, P_{n-1}\left(\phi_{\widetilde{v}^{q}}\right), P_{n}\left(\phi_{\widetilde{v}^{q}}\right), E\left(\phi_{\widetilde{v}^{q}}\right)\right)=(0, \ldots, 0, \\
\left.\sum_{\underline{m} \in \widetilde{M}(q)} \frac{\left(\beta+a_{n-1}\right) ! x_{1}^{q-\sum_{i \neq n-1} a_{i} m_{i}+a_{n-1}\left(m_{0}+1\right)} x_{2}^{m_{2}} \cdots x_{n-2}^{m_{n-2}} x_{n-1}^{-1} x_{n}^{m_{n}}}{m_{2} ! \cdots m_{n-2} ! m_{n} !\left(q-\sum_{i \neq n-1} a_{i} m_{i}+a_{n-1}\left(m_{0}+1\right)\right) !}, 0,0\right)
\end{gathered}
$$

in

$$
\frac{\left(\mathcal{O}_{X \mid Y}\right)_{p}^{n}}{\operatorname{Im}\left(\psi_{0}^{*}, \mathcal{O}_{X \mid Y}\right)_{p}}
$$

where

$\widetilde{M}(q)=\left\{\left(m_{2}, \ldots, m_{n-2}, m_{n}\right) \in \mathbb{N}^{n-2} \mid \sum a_{i} m_{i} \leq q+a_{n-1}\left(m_{0}+1\right)=\beta+a_{n-1}\right\}$

is a finite set (with $m_{0}=\frac{\beta-q}{a_{n-1}} \in \mathbb{N}$ ) and $\psi_{0}^{*}$ being the dual map of

$$
\begin{aligned}
\psi_{0}: \mathcal{D}^{n} & \longrightarrow \mathcal{D}, \\
\left(Q_{1}, \ldots, Q_{n}\right) & \mapsto \sum_{j=2}^{n} Q_{j} P_{j}+Q_{n} E .
\end{aligned}
$$


Proof. This follows from the proof of Theorem 4.23 since $\mathcal{E} x t^{1}\left(\mathcal{M}_{A}(\beta), \mathcal{O}_{X \mid Y}\right)_{p} \simeq \mathbb{C}$ for all $p \in Y \backslash Z$ and moreover

$$
\left(P_{2}\left(\phi_{v^{j}}\right), \ldots, P_{n}\left(\phi_{v^{j}}\right), E\left(\phi_{v^{j}}\right)\right)=\underline{0}
$$

for $0 \leq j \leq a_{n-1}-1, j \neq q$.

Remark 4.25. We can also compute the holomorphic solutions of $\mathcal{M}_{A}(\beta)$ at any point in $X \backslash Y$ for $A=\left(\begin{array}{llll}1 & a_{2} \ldots a_{n}\end{array}\right)$ with $1<a_{2}<\ldots<a_{n}$ and for any $\beta \in \mathbb{C}$, where $Y=\left(x_{n}=0\right) \subset X=\mathbb{C}^{n}$. We consider the vectors $w^{j}=\left(j, 0, \ldots, 0, \frac{\beta-j}{a_{n}}\right) \in$ $\mathbb{C}^{n}, j=0,1, \ldots, a_{n}-1$. Then the germs at $p \in X \backslash Y$ of the series solutions $\left\{\phi_{w^{j}}: j=0,1, \ldots, a_{n}-1\right\}$ form a basis of $\mathcal{E} x t_{\mathcal{D}}^{i}\left(\mathcal{M}_{A}(\beta), \mathcal{O}_{X}\right)_{p}$.

We have summarized the main results of this section in Figure 1. Here $A=$ $\left(1 a_{2} \cdots a_{n}\right), s \geq \frac{a_{n}}{a_{n-1}}, p \in Y \backslash Z, z \in Y \cap Z, \beta_{\text {esp }} \in \mathbb{N}$ and $\beta_{\text {gen }} \notin \mathbb{N}$.

\begin{tabular}{||l|c|c|c|c|c||}
\hline$\left(z, \beta_{\text {esp }}\right)$ & $\left(p, \beta_{\text {esp }}\right)$ & \multicolumn{2}{|c||}{} & \multicolumn{2}{|c||}{} \\
\cline { 1 - 5 }$\left(z, \beta_{\text {gen }}\right)$ & $\left(p, \beta_{\text {gen }}\right)$ & $\mathcal{E} x t^{0}\left(\mathcal{M}_{A}(\beta), \mathcal{F}\right)$ & \multicolumn{2}{c}{$x t^{1}\left(\mathcal{M}_{A}(\beta), \mathcal{F}\right)$} \\
\hline \multirow{2}{*}{$\mathcal{F}=\mathcal{O}_{X \mid Y}$} & 1 & 1 & 1 & 1 \\
\cline { 2 - 5 } & 0 & 0 & 0 & 0 \\
\hline $\mathcal{F}=\mathcal{O}_{\widehat{X \mid Y}}(s)$ & 1 & $a_{n-1}$ & 1 & 0 \\
\cline { 2 - 5 } & 0 & $a_{n-1}$ & 0 & 0 \\
\hline \multirow{2}{*}{$\mathcal{F}=\mathcal{Q}_{Y}(s)$} & 0 & $a_{n-1}$ & 0 & 0 \\
\cline { 2 - 5 } & 0 & $a_{n-1}$ & 0 & 0 \\
\hline
\end{tabular}

FiguRE 1. Dimension of the germs of $\mathcal{E} x t_{\mathcal{D}_{X}}^{i}\left(\mathcal{M}_{A}(\beta), \mathcal{F}\right)$

\section{The Case of a monomial CuRVe}

Let $A=\left(a_{1} a_{2} \cdots a_{n}\right)$ be an integer row matrix with $1<a_{1}<a_{2}<\cdots<a_{n}$ and assume without loss of generality that $\operatorname{gcd}\left(a_{1}, \ldots, a_{n}\right)=1$.

In this section we will compute the dimension of the germs of the cohomology of $\operatorname{Irr}_{Y}^{(s)}\left(\mathcal{M}_{A}(\beta)\right)$ at any point in $Y=\left\{x_{n}=0\right\} \subseteq X=\mathbb{C}^{n}$ for all $\beta \in \mathbb{C}$ and $1 \leq s \leq \infty$.

We will consider the auxiliary matrix $A^{\prime}=\left(\begin{array}{llll}1 & a_{1} & \cdots & a_{n}\end{array}\right)$ and the corresponding hypergeometric ideal $H_{A^{\prime}}(\beta) \subset A_{n+1}$, where $A_{n+1}$ is the Weyl algebra of linear differential operators with coefficients in the polynomial ring $\mathbb{C}\left[x_{0}, x_{1}, \ldots, x_{n}\right]$. We denote by $\partial_{0}$ the partial derivative with respect to $x_{0}$.

In this section we let $X^{\prime}=\mathbb{C}^{n+1}$ and we identify $X=\mathbb{C}^{n}$ with the hyperplane $\left(x_{0}=0\right)$ in $X^{\prime}$. If $\mathcal{D}_{X^{\prime}}$ is the sheaf of linear differential operators with holomorphic coefficients in $X^{\prime}$, then the analytic hypergeometric system associated with $\left(A^{\prime}, \beta\right)$, denoted by $\mathcal{M}_{A^{\prime}}(\beta)$, is by definition the quotient of $\mathcal{D}_{X^{\prime}}$ by the sheaf of ideals generated by the hypergeometric ideal $H_{A^{\prime}}(\beta) \subset A_{n+1}$ (see Section 3 ).

One of the main results in this section is the following.

Theorem 5.1. Let $A^{\prime}=\left(\begin{array}{llll}1 & a_{1} & \cdots & a_{n}\end{array}\right)$ be an integer row matrix with $1<a_{1}<$ $\cdots<a_{n}$ and $\operatorname{gcd}\left(a_{1}, \ldots, a_{n}\right)=1$. For each $\beta \in \mathbb{C}$ there exists $\beta^{\prime} \in \mathbb{C}$ such that the restriction of $\mathcal{M}_{A^{\prime}}(\beta)$ to $X=\left\{x_{0}=0\right\} \subset X^{\prime}$ is the $\mathcal{D}_{X}$-module

$$
\mathcal{M}_{A^{\prime}}(\beta)_{\mid X}:=\frac{\mathcal{D}_{X^{\prime}}}{\mathcal{D}_{X^{\prime}} H_{A^{\prime}}(\beta)+x_{0} \mathcal{D}_{X^{\prime}}} \simeq \mathcal{M}_{A}\left(\beta^{\prime}\right),
$$

where $A=\left(\begin{array}{llll}a_{1} & a_{2} & \cdots & a_{n}\end{array}\right)$. Moreover, for all but finitely many $\beta$ we have $\beta^{\prime}=\beta$. 
Proof. Following [30, we will use the notation defined in Notation4.5] and Definition 4.7. For $i=1,2, \ldots, n$ let us consider $\delta_{i} \in \mathbb{N}$ to be the smallest integer satisfying $1+\delta_{i} a_{i} \in \sum_{j \neq i} a_{j} \mathbb{N}$. Such a $\delta_{i}$ exists because $\operatorname{gcd}\left(a_{1}, \ldots, a_{n}\right)=1$.

Let us consider $\rho_{i j} \in \mathbb{N}$ such that

$$
1+\delta_{i} a_{i}=\sum_{j \neq i} \rho_{i j} a_{j}
$$

Then the operator $Q_{i}:=\partial_{0} \partial_{i}^{\delta_{i}}-\partial^{\rho_{i}}$ belongs to $I_{A^{\prime}}$, where $\partial^{\rho_{i}}=\prod_{j \neq 0, i} \partial_{j}^{\rho_{i j}}$. Moreover, for $\omega=(1,0, \ldots, 0) \in \mathbb{N}^{n+1}$ we have $\operatorname{in}_{(-\omega, \omega)}\left(Q_{i}\right)=\partial_{0} \partial_{i}^{\delta_{i}} \in \operatorname{in}_{\omega} I_{A^{\prime}}$ for $i=1, \ldots, n$.

We also have that $P_{1}=\partial_{0}^{a_{1}}-\partial_{1} \in I_{A^{\prime}}$ and $\operatorname{in}_{(-\omega, \omega)} P_{1}=\partial_{0}^{a_{1}} \in \operatorname{in}_{\omega} I_{A^{\prime}}$. Then

$$
\operatorname{in}_{\omega} I_{A^{\prime}} \supseteq\left\langle\partial_{0}^{a_{1}}, \partial_{0} \partial_{1}^{\delta_{1}} \ldots, \partial_{0} \partial_{n}^{\delta_{n}}, T_{1}, \ldots, T_{r}\right\rangle
$$

for any binomial generating system $\left\{T_{1}, \ldots, T_{r}\right\} \subseteq \mathbb{C}\left[\partial_{1}, \ldots, \partial_{n}\right]$ of the ideal $I_{A}=$ $I_{A^{\prime}} \cap \mathbb{C}\left[\partial_{1}, \ldots, \partial_{n}\right]$ (notice that $u \in L_{A}=\operatorname{ker}_{\mathbb{Z}}(A) \subset \mathbb{Z}^{n} \Longleftrightarrow(0, u) \in L_{A^{\prime}}=$ $\left.\operatorname{ker}_{\mathbb{Z}}\left(A^{\prime}\right) \subset \mathbb{Z}^{n+1}\right)$.

Using (5.1) we can prove (similarly to the proof of Lemma 4.6 for $k=1$ ) that for $\beta \notin \mathbb{N}^{*}$ or $\beta \in \mathbb{N}^{*}$ big enough, we have

$$
\partial_{0} \in \operatorname{fin}_{\omega}\left(H_{A^{\prime}}(\beta)\right)=\operatorname{in}_{\omega} I_{A^{\prime}}+\left\langle E^{\prime}\right\rangle,
$$

where $E^{\prime}=E+x_{0} \partial_{0}$ and $E:=E(\beta)=\sum_{i=1}^{n} a_{i} x_{i} \partial_{i}-\beta$. So there exists $R \in H_{A^{\prime}}(\beta)$ such that $\partial_{0}=\operatorname{in}_{(-\omega, \omega)}(R)$. In particular we have

$$
\left\langle H_{A}(\beta), \partial_{0}\right\rangle \subseteq \operatorname{fin}_{\omega}\left(H_{A^{\prime}}(\beta)\right) \subseteq \operatorname{in}_{(-\omega, \omega)}\left(H_{A^{\prime}}(\beta)\right)
$$

and the $b$-function of $H_{A^{\prime}}(\beta)$ with respect to $\omega$ is $b(\tau)=\tau$. So the restriction of $\mathcal{M}_{A^{\prime}}(\beta)$ to $\left(x_{0}=0\right)$ is a cyclic $\mathcal{D}_{X}$-module (see [30, Algorithm 5.2.8]).

In order to compute $\mathcal{M}_{A^{\prime}}(\beta)_{\mid\left(x_{0}=0\right)}$ we will follow [30, Algorithm 5.2.8]. First of all we need to describe the form of a Groebner basis of $H_{A^{\prime}}(\beta)$ with respect to $\omega$. Let $\left\{T_{1}, \ldots, T_{r}, R_{1}, \ldots, R_{\ell}\right\}$ be a Groebner basis of $I_{A^{\prime}}$ with respect to $\omega$. So we have

$$
I_{A^{\prime}}=\left\langle T_{1}, \ldots, T_{r}, R_{1}, \ldots, R_{\ell}\right\rangle
$$

and

$$
\operatorname{in}_{\omega} I_{A^{\prime}}=\left\langle T_{1}, \ldots, T_{r}, \operatorname{in}_{(-\omega, \omega)} R_{1}, \ldots, \operatorname{in}_{(-\omega, \omega)} R_{\ell}\right\rangle .
$$

If, for some $i=0, \ldots, \ell$, the $\omega$-order of $\operatorname{in}_{(-\omega, \omega)} R_{i}$ is 0 , then $\operatorname{in}_{(-\omega, \omega)} R_{i}=R_{i} \in$ $I_{A^{\prime}} \cap \mathbb{C}\left[\partial_{1}, \ldots, \partial_{n}\right]=I_{A}$ and then $\operatorname{in}_{(-\omega, \omega)} R_{i}=R_{i} \in\left\langle T_{1}, \ldots, T_{r}\right\rangle$.

If the $\omega$-order of $\operatorname{in}_{(-\omega, \omega)} R_{i}$ is greater than or equal to 1 , then $\partial_{0}$ divides $\operatorname{in}_{(-\omega, \omega)} R_{i}$. Then, according to (5.2), for $\beta \notin \mathbb{N}^{*}$ or $\beta \in \mathbb{N}^{*}$ big enough, we have

$$
\operatorname{fin}_{\omega}\left(H_{A^{\prime}}(\beta)\right)=\left\langle\partial_{0}, E, T_{1}, \ldots, T_{r}\right\rangle=\left\langle\partial_{0}\right\rangle+A_{n+1} H_{A}(\beta) \subseteq \operatorname{in}_{(-\omega, \omega)}\left(H_{A^{\prime}}(\beta)\right) .
$$

From [30, Th. 3.1.3], for all but finitely many $\beta \in \mathbb{C}$, we have

$$
\operatorname{in}_{(-\omega, \omega)}\left(H_{A^{\prime}}(\beta)\right)=\left\langle\partial_{0}, E, T_{1}, \ldots, T_{r}\right\rangle=\left\langle\partial_{0}\right\rangle+A_{n+1} H_{A}(\beta) .
$$

So, for all but finitely many $\beta \in \mathbb{C}$, the set

$$
\mathcal{G}=\left\{R, R_{1}, \ldots, R_{\ell}, E^{\prime}, T_{1}, \ldots, T_{r}\right\}
$$

is a Groebner basis of $H_{A^{\prime}}(\beta)$ with respect to $\omega$, since first of all $\mathcal{G}$ is a generating system of $H_{A^{\prime}}(\beta)$ and on the other hand $\operatorname{in}_{(-\omega, \omega)}\left(H_{A^{\prime}}(\beta)\right)=A_{n+1} \operatorname{in}_{(-\omega, \omega)}(\mathcal{G})$. 
We can now follow [30, Algorithm 5.2.8], as in the proof of Theorem4.3, to prove the result for all but finitely many $\beta \in \mathbb{C}$. Then, to finish the proof it is enough to apply Proposition 4.11 for $A^{\prime}$.

Remark 5.2. Recall that $Y=\left(x_{n}=0\right) \subset X=\mathbb{C}^{n}$ and $Z=\left(x_{n-1}=0\right) \subset X$. Let us denote $Y^{\prime}=\left\{x_{n}=0\right\} \subset X^{\prime}, Z^{\prime}=\left\{x_{n-1}=0\right\} \subset X^{\prime}$. Notice that $Y=Y^{\prime} \cap X$ and $Z=Z^{\prime} \cap X$.

By using the Cauchy-Kovalevskaya Theorem for Gevrey series (see [20, Cor. 2.2.4]), [5, Proposition 4.2] and Theorem 5.1, we get, for all but finitely many $\beta \in \mathbb{C}$ and for all $1 \leq s \leq \infty$, the following isomorphism:

$$
\mathbb{R} \mathcal{H o m}_{\mathcal{D}_{X^{\prime}}}\left(\mathcal{M}_{A^{\prime}}(\beta), \mathcal{O}_{\widehat{X^{\prime} \mid Y^{\prime}}}(s)\right)_{\mid X} \stackrel{\simeq}{\rightarrow} \mathbb{R} \mathcal{H o m}_{\mathcal{D}_{X}}\left(\mathcal{M}_{A}(\beta), \mathcal{O}_{\widehat{X \mid Y}}(s)\right)
$$

We also have the following.

Theorem 5.3. Let $A=\left(\begin{array}{llll}a_{1} & a_{2} & \cdots & a_{n}\end{array}\right)$ be an integer row matrix with $1<a_{1}<$ $a_{2}<\cdots<a_{n}$ and $\operatorname{gcd}\left(a_{1}, \ldots, a_{n}\right)=1$. Then for all $\beta \in \mathbb{C}$ we have

i) $\mathcal{E}_{x} t_{\mathcal{D}_{X}}^{0}\left(\mathcal{M}_{A}(\beta), \mathcal{Q}_{Y}(s)\right)=0$ for $1 \leq s<a_{n} / a_{n-1}$.

ii) $\mathcal{E}_{x} t_{\mathcal{D}_{X}}^{0}\left(\mathcal{M}_{A}(\beta), \mathcal{Q}_{Y}(s)\right)_{\mid Y \cap Z}=0$ for $1 \leq s \leq \infty$.

iii) $\operatorname{dim}_{\mathbb{C}}\left(\mathcal{E} x t_{\mathcal{D}_{X}}^{0}\left(\mathcal{M}_{A}(\beta), \mathcal{Q}_{Y}(s)\right)_{p}\right)=a_{n-1}$ for $a_{n} / a_{n-1} \leq s \leq \infty$ and $p \in$ $Y \backslash Z$.

iv) $\mathcal{E}_{x} t_{\mathcal{D}_{X}}^{i}\left(\mathcal{M}_{A}(\beta), \mathcal{Q}_{Y}(s)\right)=0$, for $i \geq 1$ and $1 \leq s \leq \infty$.

Here $Y=\left(x_{n}=0\right) \subset \mathbb{C}^{n}$ and $Z=\left(x_{n-1}=0\right) \subset \mathbb{C}^{n}$.

Proof. This follows from Remark 5.2, Theorem 4.1 and Proposition 4.11.

Remark 5.4. With the notation of Theorem 5.3 a basis of the $\mathbb{C}$-vector space $\mathcal{E}_{x} t_{\mathcal{D}}^{0}\left(\mathcal{M}_{A}(\beta), \mathcal{Q}_{Y}(s)\right)_{p}$ for any $\frac{a_{n}}{a_{n-1}} \leq s \leq \infty, p \in Y \backslash Z$ and $\beta \in \mathbb{C}$ is given by the "substitution" (in a sense to be made precise) of $x_{0}=0$ in the basis of $\mathcal{E} x t_{\mathcal{D}^{\prime}}^{0}\left(\mathcal{M}_{A^{\prime}}(\beta), \mathcal{Q}_{Y^{\prime}}(s)\right)_{(0, p)}$ described in Theorem 4.23.

Recall that for $A^{\prime}=\left(\begin{array}{llll}1 & a_{1} & \ldots & a_{n}\end{array}\right)$ and $\beta \in \mathbb{C}$ the $\Gamma$-series described in Section 4 are

$$
\phi_{v^{j}}=\left(x^{\prime}\right)^{v^{j}} \sum_{\substack{m_{1}, \ldots, m_{n-1}, m_{n} \geq 0 \\ \sum_{i \neq n-1} a_{i} m_{i} \leq j+a_{n-1} m_{n-1}}} \Gamma\left[v^{j} ; u(\mathbf{m})\right]\left(x^{\prime}\right)^{u(\mathbf{m})},
$$

where $x^{\prime}=\left(x_{0}, x_{1}, \ldots, x_{n}\right), \quad v^{j}=\left(j, 0, \ldots, 0, \frac{\beta-j}{a_{n-1}}, 0\right) \in \mathbb{C}^{n+1}$ for $j=0,1, \ldots$, $a_{n-1}-1$ and for $\mathbf{m}=\left(m_{1}, \ldots, m_{n}\right) \in \mathbb{Z}^{n}$ we have

$$
\left(x^{\prime}\right)^{u(\mathbf{m})}=x_{0}^{-\sum_{i \neq n-1} a_{i} m_{i}+a_{n-1} m_{n-1}} x_{1}^{m_{1}} \cdots x_{n-2}^{m_{n-2}} x_{n-1}^{-m_{n-1}} x_{n}^{m_{n}} .
$$

For $\mathbf{m}=\left(m_{1}, \ldots, m_{n}\right) \in \mathbb{N}^{n}$ such that $j-\sum_{i \neq n-1} a_{i} m_{i}+a_{n-1} m_{n-1} \geq 0$ we have

$$
\Gamma\left[v^{j} ; u(\mathbf{m})\right]=\frac{\left(\frac{\beta-j}{a_{n-1}}\right)_{m_{n-1}} j !}{m_{1} ! \cdots m_{n-2} ! m_{n} !\left(j-\sum_{i \neq n-1} a_{i} m_{i}+a_{n-1} m_{n-1}\right) !} .
$$

Since $I_{A}=I_{A^{\prime}} \cap \mathbb{C}\left[\partial_{1}, \ldots, \partial_{n}\right]$, if $I_{A^{\prime}}(f)=0$, then $I_{A}\left(f_{\mid x_{0}=0}\right)=0$ for every formal power series $f \in \mathcal{O}_{\widehat{X^{\prime} \mid Y^{\prime}}, p^{\prime}}$, where $p^{\prime}=(0, p) \in Y^{\prime} \cap\left\{x_{0}=0\right\}=Y$.

Furthermore, a Laurent monomial $\left(x^{\prime}\right)^{w^{\prime}}$ is annihilated by the Euler operator associated with $\left(A^{\prime}, \beta\right)$ if and only if $A^{\prime} w^{\prime}=\beta$ and after the substitution $x_{0}=0$ this monomial becomes zero or $x^{w}$ (in the case $w^{\prime}=(0, w)$ ) which are both annihilated 
by the Euler operator associated with $(A, \beta)$, since $A w=A^{\prime} w^{\prime}=\beta$ in the case $w^{\prime}=(0, w)$.

Hence, for $p \in Y$, every formal series solution $f \in \mathcal{O}_{\widehat{X^{\prime} \mid Y^{\prime}},(0, p)}$ of $\mathcal{M}_{A^{\prime}}(\beta)$ becomes, after the substitution $x_{0}=0$, a formal series solution $f_{\mid x_{0}=0} \in \mathcal{O}_{\widehat{X \mid Y}, p}$ of $\mathcal{M}_{A}(\beta)$. The analogous result is also true for convergent series solutions at a point of $x_{0}=0$.

After the substitution $x_{0}=0$ in the series $\phi_{v^{j}}$ we get

$$
\phi_{v^{j} \mid x_{0}=0}=\sum_{\substack{m_{1}, \ldots, m_{n-1}, m_{n} \geq 0 \\ \sum a_{i} m_{i}=j+a_{n-1} m_{n-1}}} \frac{\left(\frac{\beta-j}{a_{n-1}}\right)_{m_{n-1}} j ! x_{1}^{m_{1}} \cdots x_{n-2}^{m_{n-2}} x_{n-1}^{\frac{\beta-j}{a_{n-1}}-m_{n-1}} x_{n}^{m_{n}}}{m_{1} ! \cdots m_{n-2} ! m_{n} !}
$$

for $j=0,1, \ldots, a_{n-1}-1$.

The summation before is taken over the set

$$
\Delta_{j}:=\left\{\left(m_{1}, \ldots, m_{n}\right) \in \mathbb{N}^{n}: \sum_{i \neq n-1} a_{i} m_{i}=j+a_{n-1} m_{n-1}\right\} .
$$

It is clear that $(0, \ldots, 0) \in \Delta_{0}$ and for $j \geq 1, \Delta_{j}$ is a nonempty set since $\operatorname{gcd}\left(a_{1}, \ldots, a_{n}\right)=1$. Moreover $\Delta_{j}$ is a countably infinite set for $j \geq 0$. To this end take some $\underline{\lambda}:=\left(\lambda_{1}, \ldots, \lambda_{n}\right) \in \Delta_{j}$. Then $\underline{\lambda}+\mu\left(0, \ldots, 0, a_{n}, a_{n-1}\right)$ is also in $\Delta_{j}$ for all $\mu \in \mathbb{N}$.

The series $\phi_{v^{j} \mid x_{0}=0}$ is a Gevrey series of order $s=\frac{a_{n}}{a_{n-1}}$ since $\phi_{v^{j}}$ is also. We will see that in fact the Gevrey index of $\phi_{v^{j} \mid x_{0}=0}$ is $\frac{a_{n}}{a_{n-1}}$ for $j=0, \ldots, a_{n-1}-1$ such that $\frac{\beta-j}{a_{n-1}} \notin \mathbb{N}$. To this end let us consider the subsum of $\phi_{v^{j} \mid x_{0}=0}$ over the set of $\left(m_{1}, \ldots, m_{n}\right) \in \mathbb{N}^{n}$ of the form $\underline{\lambda}^{(j)}+\mathbb{N}\left(0, \ldots, 0, a_{n}, a_{n-1}\right)$ for some fixed $\underline{\lambda}^{(j)} \in \Delta_{j}$. Then we get the series:

$$
\frac{j !\left(\frac{\beta-j}{a_{n-1}}\right)_{\lambda_{n-1}^{(j)}} x_{1}^{\lambda_{1}^{(j)}} \cdots x_{n-2}^{\lambda_{n-2}^{(j)}} x_{n-1}^{\frac{\beta-j}{a_{n-1}}-\lambda_{n-1}^{(j)}}}{\lambda_{1}^{(j)} ! \cdots \lambda_{n-2}^{(j)} !} \sum_{m \geq 0} \frac{\left(\frac{\beta-j}{a_{n-1}}-\lambda_{n-1}^{(j)}\right)_{a_{n} m} x_{n-1}^{-a_{n} m} x_{n}^{\lambda_{n}^{(j)}+a_{n-1} m}}{\left(\lambda_{n}^{(j)}+a_{n-1} m\right) !}
$$

and it can be proven, by using the d'Alembert ratio test, that its Gevrey index equals $\frac{a_{n}}{a_{n-1}}$ at any point in $Y \backslash Z$, for any $j=0, \ldots, a_{n-1}-1$ such that $\frac{\beta-j}{a_{n-1}} \notin \mathbb{N}$.

For all $j=0, \ldots, a_{n-1}-1$ we have

$$
\phi_{v^{j} \mid x_{0}=0} \in x_{n-1}^{\frac{\beta-j}{a_{n-1}}} \mathbb{C}\left[\left[x_{1}, \ldots, x_{n-2}, x_{n-1}^{-1}, x_{n}\right]\right]
$$

and in particular these $a_{n-1}$ series are linearly independent and hence a basis of $\mathcal{E}_{x} t^{0}\left(\mathcal{M}_{A}(\beta), \mathcal{O}_{\widehat{X \mid Y}}(s)\right)_{p}$ for $s \geq a_{n} / a_{n-1}$ and $\left.p \in Y \backslash Z\right)$ if all of them are nonzero (see Theorem 4.21).

If there exists $0 \leq j \leq a_{n-1}-1$ such that $\phi_{v^{j} \mid x_{0}=0}=0$, then we have that $\phi_{v^{j}}$ is a polynomial divisible by $x_{0}$ and this happens if and only if $\beta \in \mathbb{N} \backslash \mathbb{N} A$.

In this last case we do not get a basis of $\mathcal{E} x t^{0}\left(\mathcal{M}_{A}(\beta), \mathcal{O}_{\widehat{X \mid Y}}(s)\right)_{p}$ by the previous procedure. We will proceed as follows. Let us consider $w^{\prime}=(0, \omega) \in \mathbb{N}^{n+1}$ such that $\beta^{\prime}:=\beta-A^{\prime} w^{\prime} \in \mathbb{Z}_{<0}$. Then taking the basis $\left\{\phi_{v^{j}} \mid j=0, \ldots, a_{n-1}-1\right\}$ of $\mathcal{E} x t^{0}\left(\mathcal{M}_{A^{\prime}}\left(\beta^{\prime}\right), \mathcal{O}_{\widehat{X^{\prime} \mid Y^{\prime}}}(s)\right)_{(0, p)}$ given by Theorem 4.21 and after the substitution $x_{0}=0$, we get a basis of $\mathcal{E} x t^{0}\left(\mathcal{M}_{A}\left(\beta^{\prime}\right), \mathcal{O}_{\widehat{X \mid Y}}(s)\right)_{p}$ for $s \geq a_{n} / a_{n-1}$ and $p \in Y \backslash Z$. 
Since $\beta, \beta^{\prime} \in \mathbb{Z} \backslash \mathbb{N} A$, then $\partial^{w}: \mathcal{M}_{A}\left(\beta^{\prime}\right) \rightarrow \mathcal{M}_{A}(\beta)$ is an isomorphism (see [31, Remark 3.6] and [4, Lemma 6.2]) and we can use this isomorphism to obtain a basis of $\mathcal{E}_{x} t^{0}\left(\mathcal{M}_{A}(\beta), \mathcal{O}_{\widehat{X \mid Y}}(s)\right)_{p}$.

Using the previous discussion and ideas similar to the ones of Section 4 (we will use the notation therein) we can prove the following theorem.

Theorem 5.5. Let $A=\left(\begin{array}{llll}a_{1} & a_{2} & \cdots & a_{n}\end{array}\right)$ be an integer row matrix with $0<a_{1}<$ $a_{2}<\cdots<a_{n}, Y=\left(x_{n}=0\right) \subset X$ and $Z=\left(x_{n-1}=0\right) \subset X$. Then for all $p \in Y \backslash Z, \beta \in \mathbb{C}$ and $s \geq a_{n} / a_{n-1}$ we have:

i) If $\beta \notin \mathbb{N}$, then:

$$
\mathcal{E}_{x} t^{0}\left(\mathcal{M}_{A}(\beta), \mathcal{Q}_{Y}(s)\right)_{p}=\bigoplus_{j=0}^{a_{n-1}-1} \mathbb{C} \overline{\left(\phi_{v^{j} \mid x_{0}=0}\right)_{p}} .
$$

ii) If $\beta \in \mathbb{N}$, then there exists a unique $q \in\left\{0, \ldots, a_{n-1}-1\right\}$ such that $\frac{\beta-q}{a_{n-1}} \in \mathbb{N}$ and we have:

$$
\mathcal{E}_{x} t^{0}\left(\mathcal{M}_{A}(\beta), \mathcal{Q}_{Y}(s)\right)_{p}=\bigoplus_{q \neq j=0}^{a_{n-1}-1} \mathbb{C} \overline{\left(\phi_{v^{j} \mid x_{0}=0}\right)_{p}} \oplus \mathbb{C} \overline{\left(\phi_{\widetilde{v^{q}} \mid x_{0}=0}\right)_{p}} .
$$

Here $\bar{\phi}$ stands for the class modulo $\mathcal{O}_{X \mid Y, p}$ of $\phi \in \mathcal{O}_{\widehat{X \mid Y}, p}(s)$.

Remark 5.6. We can also compute the holomorphic solutions of $\mathcal{M}_{A}(\beta)$ at any point in $X \backslash Y$ for $A=\left(a_{1} a_{2} \ldots a_{n}\right)$ with $0<a_{1}<a_{2}<\ldots<a_{n}$ and for any $\beta \in \mathbb{C}$, where $Y=\left(x_{n}=0\right) \subset X=\mathbb{C}^{n}$ (see [7, Sec. 2.1] and Remark 4.25). As in the beginning of Section 5 let us consider the auxiliary matrix $A^{\prime}=\left(\begin{array}{lllll}1 & a_{1} & a_{2} & \ldots & a_{n}\end{array}\right)$ and the notation therein.

Let us consider the vectors $w^{j}=\left(j, 0, \ldots, 0, \frac{\beta-j}{a_{n}}\right) \in \mathbb{C}^{n+1}, j=0,1, \ldots, a_{n}-1$. Then the germs at $p^{\prime}=(0, p) \in X^{\prime} \backslash Y^{\prime}$ (with $p \in X \backslash Y$ ) of the series solutions $\left\{\phi_{w^{j}}: j=0,1, \ldots, a_{n}-1\right\}$ form a basis of $\mathcal{E} x t_{\mathcal{D}^{\prime}}^{0}\left(\mathcal{M}_{A^{\prime}}(\beta), \mathcal{O}_{X^{\prime}}\right)_{p^{\prime}}$. Taking

$$
\left\{\phi_{w^{j} \mid x_{0}=0}: j=0,1, \ldots, a_{n}-1\right\}
$$

we get a basis of $\mathcal{E} x t_{\mathcal{D}}^{0}\left(\mathcal{M}_{A}(\beta), \mathcal{O}_{X}\right)_{p}$ for $\beta \in \mathbb{C}$ such that $\beta \notin \mathbb{N} \backslash \mathbb{N} A$ at any point $p \in X \backslash Y$. When $\beta \in \mathbb{N} \backslash \mathbb{N} A$ we can proceed as in Remark 5.4.

\section{Conclusions}

1) In Sections 4 and 5 we have proved that the irregularity complex $\operatorname{Irr}_{Y}^{(s)}\left(\mathcal{M}_{A}(\beta)\right)$ is zero for $1 \leq s<a_{n} / a_{n-1}$ and concentrated in degree 0 for $a_{n} / a_{n-1} \leq s \leq \infty$ (see Theorems 4.1 and 5.3). Here $A$ is a row integer matrix $\left(a_{1} a_{2} \cdots a_{n}\right)$ with $0<a_{1}<a_{2}<\cdots<a_{n}$ and $\beta$ is a parameter in $\mathbb{C}$. We have reduced the case $a_{1}>1$ to the one where $a_{1}=1$ and then to the two-dimensional case treated in [7].

2) We have described a basis of $\mathcal{E}_{x} t_{\mathcal{D}_{X}}^{0}\left(\mathcal{M}_{A}(\beta), \mathcal{Q}_{Y}(s)\right)_{p}$ for $p \in Y \backslash Z$ and $a_{n} / a_{n-1} \leq s \leq \infty$ (see Theorems 4.23 and 5.5). Here $Y=\left(x_{n}=0\right) \subset X=\mathbb{C}^{n}$ and $Z=\left(x_{n-1}=0\right) \subset X$. From the form of the basis it is easy to see that the eigenvalues of the corresponding monodromy, with respect to $Z$, are simply $\exp \left(\frac{2 \pi i(\beta-k)}{a_{n-1}}\right)$ for $k=0, \ldots, a_{n-1}-1$. Notice that for $\beta \in \mathbb{Z}$ one eigenvalue (the one corresponding to the unique $k=0, \ldots, a_{n-1}-1$ such that $\left.\frac{\beta-k}{a_{n-1}} \in \mathbb{Z}\right)$ is just 1 . See Remark 5.4 for the notation. 


\section{REFERENCES}

[1] Adolphson, A. Hypergeometric functions and rings generated by monomials. Duke Math. J. 73 (1994), no. 2, 269-290. MR.1262208 (96c:33020)

[2] Assi, A., Castro-Jiménez, F. J. and Granger, J.-M. How to calculate the slopes of a D- module. Compositio Math., 104 (1996) 107-123. MR1421395(98i:32010)

[3] Beilinson, A. A., Bernstein, J. and Deligne, P. Faisceaux pervers. Analysis and topology on singular spaces, I (Luminy, 1981), 5-171, Astérisque, 100, Soc. Math. France, Paris, 1982. MR751966 (86g:32015)

[4] Berkesch, Ch. The rank of a hypergeometric system. arXiv:0807.0453v1 [math.AG].

[5] Castro-Jiménez, F.J. and Takayama, N. Singularities of the hypergeometric system associated with a monomial curve. Transactions of the American Mathematical Society, vol.355, no. 9, (2003), 3761-3775. MR1990172 (2004j:32009)

[6] Cattani, E., D'Andrea, C. and Dickenstein, A. The A-hypergeometric system associated with a monomial curve. Duke Math. J. 99 (1999), no. 2, 179-207. MR.1708034 (2001f:33018)

[7] Fernández-Fernández, M.C. and Castro-Jiménez, F.J. Gevrey solutions of irregular hypergeometric systems in two variables. arXiv:0811.3390v1 [math.AG].

[8] Gelfand, I.M., Graev, M.I. and Zelevinsky, A.V. Holonomic systems of equations and series of hypergeometric type. Dokl. Akad. Nauk SSSR 295 (1987), no. 1, 14-19; translation in Soviet Math. Dokl. 36 (1988), no. 1, 5-10. MR902936 (88j:58118)

[9] Gelfand, I.M., Zelevinsky, A.V. and Kapranov, M.M., Equations of hypergeometric type and Newton polyhedra. Dokl. Akad. Nauk SSSR 300 (1988), no. 3, 529-534; translation in Soviet Math. Dokl. 37 (1988), no. 3, 678-682. MR948812 (89j:22015)

[10] Gelfand, I.M., Zelevinsky, A.V. and Kapranov, M.M., Hypergeometric functions and toric varieties (or Hypergeometric functions and toral manifolds). Translated from Funktsional. Anal. i Prilozhen. 23 (1989), no. 2, 12-26; translation in Funct. Anal. Appl. 23 (1989), no. 2, 94-106; and I.M. Gelfand, A.V. Zelevinskiŭ and M.M. Kapranov, Correction to the paper: "Hypergeometric functions and toric varieties" [Funktsional. Anal. i Prilozhen. 23 (1989), no. 2, 12-26]; (Russian) Funktsional. Anal. i Prilozhen. 27 (1993), no. 4, 91; translation in Funct. Anal. Appl. 27 (1993), no. 4, 295 (1994). MR.1011353 (90m:22025)

[11] Hartillo, M. I. Irregular hypergeometric systems associated with a singular monomial curve. Transactions of the American Mathematical Society, vol. 357, no. 11 (2004), 4633-4646. MR2156724 (2006h:32005)

[12] Iwasaki, K. Cohomology groups for recurrence relations and contiguity relations of hypergeometric systems. J. Math. Soc. Japan 55, 2 (2003), 289-321. MR.1961288 (2004m:39031)

[13] Kashiwara, M. On the Maximally Overdetermined System of Linear Differential Equations. Publ. Res. Inst. Math. Sci., Kyoto Univ. 10 (1975), 563-579. MR0370665 (51:6891)

[14] Kashiwara, M. The Riemann-Hilbert problem for holonomic systems Publ. Res. Inst. Math. Sci., 20 (1984), 319-365. MR743382 (86j:58142)

[15] Kashiwara, M. and Kawai, T. On the holonomic systems of micro-differential equations III Publ. Res. Inst. Math. Sci., 17 (1981), 813-979. MR650216 (83e:58085)

[16] Komatsu, H. On the index of ordinary differential operators. J. Fac. Sci. Univ. Tokyo Sect. IA Math., 18 (1971), 379-398. MR0303568 (46:2705)

[17] Laurent, Y. Calcul d'indices et irrégularité pour les systèmes holonomes. Differential systems and singularities (Luminy, 1983). Astérisque No. 130 (1985), 352-364. MR804063(87a:58141)

[18] Laurent, Y. Polygone de Newton et b-fonctions pour les modules microdifférentiels. Annales scientifiques de l'Ecole Norm. Sup. $4^{e}$ série, tome 20, no. 3 (1987), 391-441. MR925721 (89k:58282)

[19] Laurent, Y. and Mebkhout, Z. Pentes algébriques et pentes analytiques d'un D-module. Annales Scientifiques de l'Ecole Norm. Sup. $4^{e}$ série, tome 32, n. 1 (1999), 39-69. MR.1670595 (2001b:32015)

[20] Laurent, Y. and Mebkhout, Z. Image inverse d'un $\mathcal{D}$-module et polygone de Newton. Compositio Math. 131 (2002), no. 1, 97-119. MR.1895922 (2003g:32021)

[21] Majima, H. Irregularities on hyperplanes of holonomic $\mathcal{D}$-module (especially defined by confluent hypergeometric partial differential equations). Complex analysis and microlocal analysis (Kyoto, 1997). Surikaisekikenkyusho Kokyuroku No. 1090 (1999), 100-109. MR:1750022 (2001g:32024) 
[22] Malgrange, B. Sur les points singuliers des équations différentielles. L'Enseignement Mathématique, XX, 1-2, (1974), 147-176. MR0368074 (51:4316)

[23] Matusevich, L.F., Miller, E. and Walther, U. Homological methods for hypergeometric families. J. Amer. Math. Soc. 18 (2005), 919-941. MR2163866 (2007d:13027)

[24] Mebkhout, Z. Une équivalence de catégories. Compositio Math. 51 (1984), no. 1, 51-62, and Une autre équivalence de catégories. Compositio Math. 51 (1984), no. 1,63-88. MR734785 (85k:58073)

[25] Mebkhout, Z. Le théorème de positivité de l'irrégularité pour les $\mathcal{D}_{X}$-modules, in The Grothendieck Festschrift, Progress in Math., vol.88, no.3, Birkhäuser (1990), 83-131. MR.1106912 (92j:32031)

[26] Oaku, T. On regular b-functions of D-modules. Preprint January 18, 2007.

[27] Ohara, K. and Takayama, N. Holonomic rank of A-hypergeometric differential-difference equations. http://arxiv.org/abs/0706.2706v1

[28] Ramis, J. P. Théorèmes d'indices Gevrey pour les équations différentielles ordinaires. Memoirs of the AMS 48, (1984), 296. MR733946 (86e:34021)

[29] Saito, M. Isomorphism classes of A-hypergeometric systems. Compositio Mathematica 128 (2001), 323-338. MR1858340 (2003f:33019)

[30] Saito, M., Sturmfels, B. and Takayama, N. Gröbner Deformations of Hypergeometric Differential Equations. Algorithms and Computation in Mathematics 6. Springer. MR 1734566 (2001i:13036)

[31] Schulze, M. and Walther, U. Hypergeometric D-modules and twisted Gauss-Manin systems. J. Algebra 322 (2009), 3392-3409. MR2567427

[32] Schulze, M. and Walther, U. Irregularity of hypergeometric systems via slopes along coordinate subspaces. Duke Math. J. 142 (2008), no. 3, 465-509. MR2412045 (2009b:13067)

[33] Takayama, N. Modified A-hypergeometric Systems. arXiv:0707.0043v2 [math.CA]

Faculty of Mathematics, Department of Algebra, University of Sevilla, Seville, SPAIN

E-mail address: mcferfer@us.es

Faculty of Mathematics, Department of Algebra, University of Sevilla, Seville, SPAIN

E-mail address: castro@us.es 